\title{
On the deviation of computed permeability induced by unresolved morphological features of the pore space
}

\author{
S. Perez • P. Moonen - P. Poncet
}

Received: 26-01-2021 / Accepted: 25-10-2021

\begin{abstract}
This article describes how much the computed absolute permeability is impacted by the slip effect at the fluid/solid interface, in the context of single-phase pore-scale flow. While this effect is well quantified in microchannels or simple geometries, the present study focuses on its average effect in real rock matrix geometries, by means of highresolution X-ray microtomography. Due to the inherently finite resolution of the technique, an uncertainty exists on the true position of the fluid/solid interface and its morphological features below the image resolution (unseen roughness). We demonstrate that both these uncertainties can be interpreted as a slip condition, and consequently we focus on how a slip length can impact the computed absolute permeability, after having provided an estimation of a meaningful bound on the slip coefficient. To that extent, two strategies are employed: the global deviation of permeability, and the theoretically established linear deviation. Three high-definition 3D geometries are used as practical examples of our methodology. Results are discussed in terms of relative deviation versus specific surface area, and lead to quantities of interest involving the linear deviation of permeability.
\end{abstract}

Keywords Pore scale modeling · Uncertainty management · Digital rock physics · Slip flows · Absolute permeability

\section{Introduction}

According to Encyclopædia Britannica, permeability is "the capacity of a porous material for transmitting fluid; it is expressed as the velocity with which a fluid of specified viscosity, under the influence of a given pressure, passes through a sample having a certain cross section and thickness" [1]. This deceivingly simple definition hides a complex reality at pore scale, even when only a single-phase fluid is involved, in particular because of the complex 3D morphology of real porous media.

The advent of X-ray micro tomography (X-ray $\mu \mathrm{CT}$ ) makes it possible to non-destructively determine such morphology of real, opaque, porous media. An X-ray scan provides a 3D data volume composed of elementary cubes, called voxels. Each voxel is characterized by a level of grey that is proportional to the local X-ray attenuation of the material. As the attenuation is material-dependent, the pore space can easily be separated from the surrounding matrix. The resulting dataset can be combined naturally with pore-scale numerical models [2, 3]. Pore-scale models of flow and transport roughly fall into one of two broad categories: (a) direct numerical simulators (DNS) and (b) pore-network models (PNM) [4]. At low Reynolds number, both solve the same fundamental governing equations, but in the former case the true geometry is employed, whereas in PNM the porous medium is regarded as a number of pores, interlinked by flow channels. Consequently, DNS based on Stokes and Navier-Stokes equations promises to be more precise, at the cost of being computationally more expensive. On the other hand, PNM models based on Hagen-Poiseuille formula are highly efficient, and their way of conceptualizing the true geometry offers a mental framework for transposing the conclusions of fundamental research on idealised flow channels, into real rock geometries.

However, limitations exist in the X-ray $\mu \mathrm{CT}$ imaging process that may affect the medium effective properties computation with such numerical methods. Actually, as shown in Figure 1 the interfaces do not occur as intensity steps in $\mu \mathrm{CT}$-datasets, but rather as a gradual intensity gradient spanning several voxels. This is due to a combination of mainly two factors.

Firstly, due to the imaging resolution the interface generally does not coincide with a voxel boundary. That is, it separates the voxel in two distinct parts, and the gray level of the voxel is intermediate to the gray levels on either side of the interface. This is the so-called partial volume effect leading to unresolved morphological features including the roughness of the pore space walls.

S. Perez and P. Poncet

Universite de Pau et des Pays de l'Adour, E2S UPPA, CNRS, LMAP, Pau, France E-mail: philippe.poncet@univ-pau.fr

P. Moonen

Universite de Pau et des Pays de l'Adour, E2S UPPA, CNRS, Total, LFCR, Pau, France

Universite de Pau et des Pays de l'Adour, E2S UPPA, CNRS, DMEX, Pau, France 


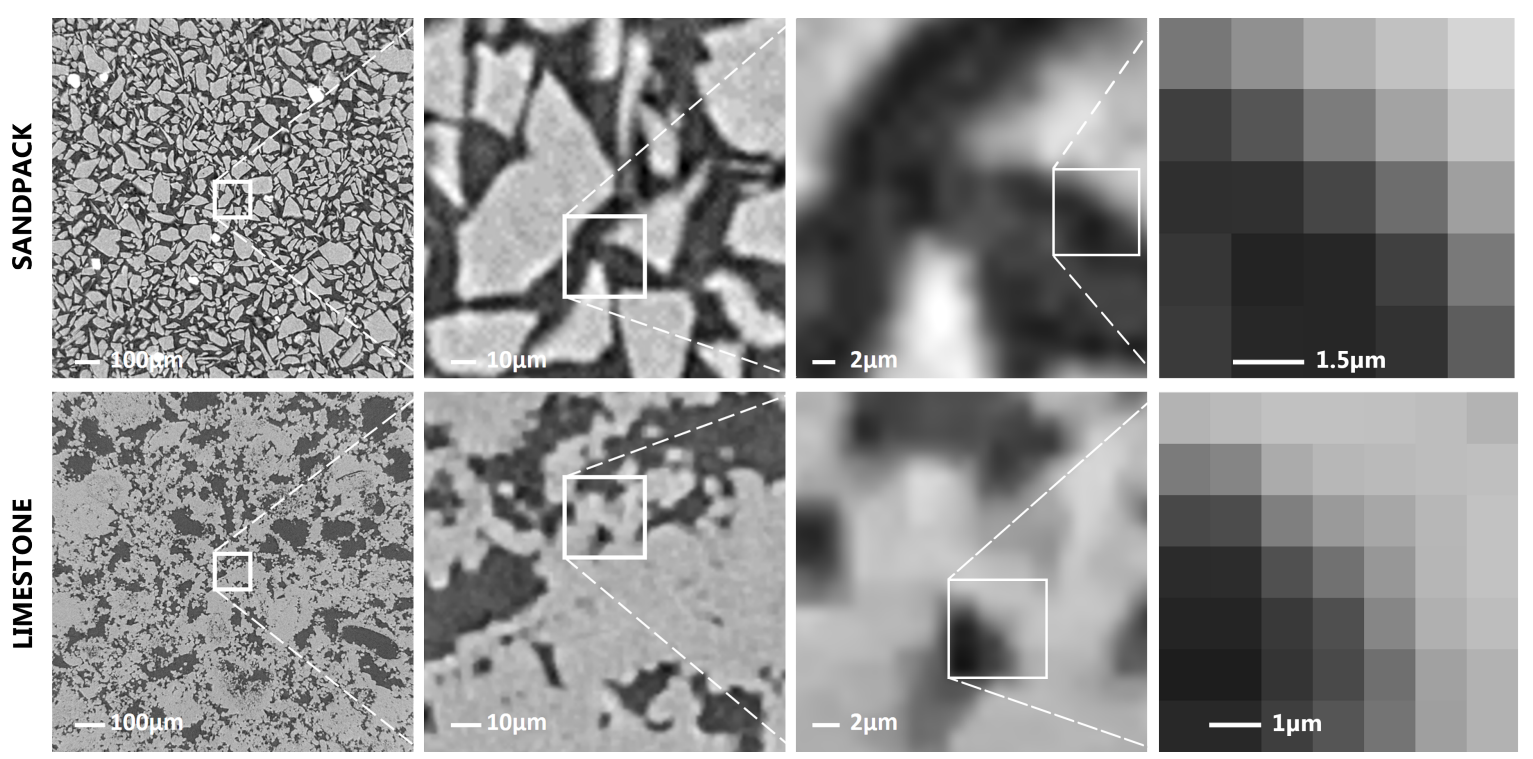

Fig. 1 High resolution X-ray scans of two samples. Data are numerically magnified up to voxel scale using interpolated zoom up to the final zoom on the right, at the physical size of the pixel (voxel sizes are respectively $1.5 \mu \mathrm{m}$ and $1 \mu \mathrm{m}$ for the sandpack and the limestone). This illustrates the presence of a gray-scale gradient instead of a sharp interface. The sandpack is the geometry used in section 4.4.

Secondly, the finite resolution of the imaging pipeline thus blurs the material interfaces over a width of multiple voxels [5]. In the presence of sharp density transitions, the different refraction index on either side of the interface leads to so-called edge enhancement which manifests itself as an over- and undershoot of the gray level immediately next to the interface [6]. It means that beside the approximation based on the roughness of the pore space walls, X-ray tomography also approximates the position of those pore walls. Consequently, the "true" morphology of the pore space is unknown, and the calculation of effective properties is prone to uncertainty [7]. While these effects can be minimized, they cannot be eliminated from the imaging process. A model that directly uses the blurred interface as input therefore has a more practical interest than geometry-conforming models with an approximate pore-space morphology.

The present article addresses the question of the reliability of the permeability value induced by the uncertainties introduced in the two last paragraphs, both mathematically translating into models of slip flows. The unseen roughness of a channel wall and the exact position of that wall -and hence the channel dimensions- will generally be visible only in an approximate sense on an X-ray $\mu \mathrm{CT}$ scan of a representative volume of material. This is not to say that sufficient resolution cannot be obtained, but rather that real pore media covering a wide range of pore scales generally requires a compromise between the volume being investigated and the scan resolution. The former has to be large enough to be representative, whereas the latter has to be small enough to observe enough details.

The present study thus focuses on quantifying the uncertainty on the computed permeability by providing a range of possible values instead of a single value, and accounting for the deviation between the true morphology of the pore space and the assumed one. We focus on the absolute permeability, which means that we consider the single-phase flow of a Newtonian fluid through the pore space in an impermeable rock matrix. We will employ a slip length formalism and use the variation in slip length as a way to represent the uncertainty on the geometry. While the slip formalism has been intensively studied and developed for porous media $[8,9]$, we focus on its practical aspects and use for real three-dimensional rock geometries.

This article starts with a Material and Method section 2 that begins to introduce the well-known slip formalism and the two methods considered to get the ranges of permeability values, namely the full permeability deviation and the linear permeability deviation. The governing equations, here the stationary 3D Stokes equation in the pore space involving the slip condition, are defined in section 2.2, along with the construction of the tangent and normal fields on the fluid/solid interface. The numerical schemes and their related numerical analysis, used for solving efficiently the Stokes system with slip, are detailed in the appendices A and B. Finally, the selected datasets to illustrate different features of the proposed computational method are briefly presented in section 2.3 .

In section 3, the focus is given on establishing the linear momentum $L_{0}$ by means of the asymptotic development detailed in appendix $\mathrm{C}$, and on the choice of a representative value of the slip length $\beta$ based mainly on the voxel size and adjusted when possible with the slip due to the pore roughness.

The section 4 finally investigates several types of real porous rock with very different pore structures, in order to put in practice this methodology. It begins with a validation of the numerical method (developed for this specific analysis) on a cylindrical synthetic geometry, including a convergence study and an analysis of the absolute permeability. The samples considered for this study are an unconsolidated sand pack, and two types of sandstone: a Bentheimer sandstone and Castlegate outcrop sandstone. Their voxel sizes $h$ range from $1.5 \mu m$ for the sandpack and $2.2 \mu m$ for the Bentheimer sandstone to $5.6 \mu \mathrm{m}$ for the Castlegate sandstone. A discussion on the relative deviation for representative samples, and non-dimensional quantities of interest, conclude the section and our analysis. 


\section{Material and methods}

\subsection{Slip modeling}

In an isolated flow channel, fluid flow encounters a resistance caused by viscous shear stresses which are dependent, among many things, on the roughness of the channel material. The permeability of the channel wall itself also plays a role $[10,11,12]$, with more permeable walls increasing the slip velocity on the porous wall. Furthermore, chemical effects and hydrophobic properties may also cause slip [13,14], as well as trapped gas or liquid along the pore walls [15], clearly quantified for fully wetting films [16].

Although these situations involve slip conditions illustrating physical processes, the idea of using a slip length formalism to describe flow over a rough surface is not new. Historically the relation between roughness and slip length had been proposed by Navier as early as 1823 [17] and established by computation in [18]. Nowadays, for an ideal impermeable rock matrix whose pore space is filled with a Newtonian fluid, the linear formulation of the slip, based on the Stokes equations with no-slip-through $u \cdot n=0$ at the solid boundary, is quite conventional [19, 13, 20] and its tangential components are inherited from the relation

$$
u-\beta D(u) n=0
$$

where $D(u)=\left(\nabla u+\nabla u^{T}\right) / 2$ is the shear-rate tensor, that is to say the symmetric part of the velocity gradient, $n$ is the unit vector field normal to the fluid/solid interface and directed toward the fluid region, and $\beta$ is the slip length.

Since Navier, many researchers have attempted to elucidate the precise relationship between permeability and roughness. Numerical studies have focused on various, mostly periodic, roughness patterns such as trenches (ridges, transverse or not) $[19,21,22]$, pillars [23, 24] or holes [25]. These and other studies showed that for a given kind of pattern the pitch and the liquid-solid contact area are the two most important parameters for determining the slip length, i.e. the extrapolated distance relative to the surface where the tangential velocity component vanishes [26]. Degruyter et al. [27] showed that the permeability of laminar and turbulent gas escape during the ascent of rhyolitic magma in volcanic conduits depends on the -unresolved- surface roughness. Similarly, Noiriel et al. [28] found that permeability changes are linked to changes in pore surface roughness, induced by calcite precipitation. The hydrophobic and/or slip length reported in literature ranges typically from $200 \mathrm{~nm}$ [29] to $700 \mathrm{~nm}$ for multi-phase flows [15] and even $950 \mathrm{~nm}$ [23]. Slip lengths in the range $500-860 \mathrm{~nm}$ have been observed on a mica/water interface with a roughness of $15 \mathrm{~nm}$ [13].

In brief, the uncertainties on the geometrical features of a pore space obtained through X-ray tomography, whether it is unresolved roughness or an approximate pore wall position, can be modelled by a slip length. When both uncertainties occur simultaneously, as is generally the case, Daly and Roose [30] showed by means of homogenization that the surface roughness is the key property of the microscale geometry which determines the hydraulic conductivity at the macro-scale.

Consequently, the method considered to bring a range of permeability values dealing with these uncertainties relies on the following formalism. First, it is to estimate a bulk absolute permeability $K_{0}$ thanks to solving numerically the Stokes system (3) introduced in the next section 2.2 with adherent boundary conditions, and then estimate a permeability $K_{\beta}$ using the slip condition (1) with a meaningful slip length. Such a choice of a value for a maximum slip length is developed in section 3.2, and leads to the interval $\left[K_{0}, K_{\beta}\right]$ for the computed permeability, which can be called naturally the raw permeability deviation or the full permeability deviation.

Furthermore, an other method to evaluate such interval is to consider and use the linear momentum $L_{0}$ of $K_{\beta}$ from a given material of bulk permeability $K_{0}$, introduced in the linear expansion of the permeability $K_{\beta}$ written as

$$
K_{\beta}=K_{0}+\beta L_{0}+\mathcal{O}\left(\beta^{2}\right) .
$$

The range of permeability is then given by $\left[K_{0}, K_{0}+\beta L_{0}\right]$, which can be called the linear permeability deviation, which is especially convenient when the user needs to change the value of $\beta$ or apply a different coefficient of security on it. Moreover, it could also be possible to estimate $L_{0}$ numerically by its approximation $\left(K_{\beta}-K_{0}\right) / \beta$ but this method is unreliable due to the strong noise of $K_{\beta}$ with respect to $\beta$ (see Figure 10 for the Castlegate sandstone for example). The latter approach be clarified by considering Poisson's equation on the $1 \mathrm{D}$ domain $[0, l]$ of length $l$ with a constant driving force $f$, an adherent condition in $l$ and a slip condition $u-\beta u^{\prime}=0$ in 0 . In this toy example, we easily find $K_{0}=l^{2} / 12$, its linear deviation $L_{0}=l / 4$, and a remainder scaling as $\beta^{2}$.

More generally, our aim is to provide from a representative slip value $\beta$, a range of values $\left[K_{0}, K_{\beta}\right]$ or $\left[K_{0}, K_{0}+\right.$ $\beta L_{0}$ ] for the absolute permeability, instead of a single value of $K_{0}$ whose confidence level is often questionable [31]. Each of our practical study cases is performed with a single meaningful slip length value $\beta$, since any other deviation induced by a different slip length can be resolved by linear extrapolation due to this linear feature. The method to establish the problem to solve in order to get the value of $L_{0}$ is developed in section 3.1 and detailed in appendix C.

\subsection{Governing equations}

We introduce a computational domain $\Omega$ divided into a solid part $\Omega_{S}$, representing the solid matrix of the porous medium, and a fluid part $\Omega_{F}=\Omega \backslash \overline{\Omega_{S}}$, corresponding to the pore space (Figure 2). The latter is assumed to be a smooth 


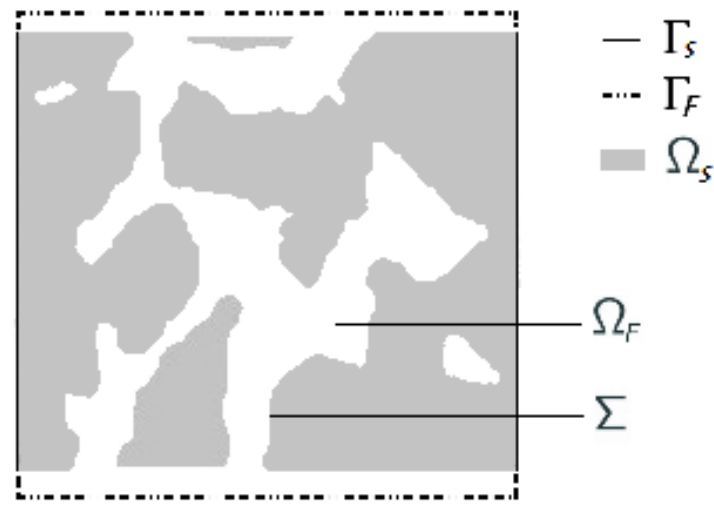

Fig. 2 General schematic of the computational domain $\Omega$. The geometry is laterally constrained by a solid layer and the top and bottom surfaces by a fluid layer to so as to be able to impose periodic boundary conditions on $\partial \Omega=\Gamma_{S} \cup \Gamma_{F}$ in the three spatial directions. The image shows some apparently unconnected pores. This is because it presents a $2 \mathrm{D}$ slice through the $3 \mathrm{D}$ connected pore network. The calculations are performed on the full $3 \mathrm{D}$ geometry.

connected open set. In practice, real rock geometries can present unconnected pores, so they do not contribute to the flow. The preceding hypothesis therefore remains valid and avoids computing a (zero) velocity field in the unconnected pores. We denote the computational domain boundary by $\partial \Omega$, and use $\Gamma_{F}=\partial \Omega \cap \Omega_{F}$ and $\Gamma_{S}=\partial \Omega \cap \Omega_{S}$ to refer to the fluid and solid parts of the computational domain boundary, respectively, such that $\partial \Omega=\Gamma_{F} \cup \Gamma_{S}$. The internal fluid/solid continuous interface is defined by $\Sigma=\partial \Omega_{F} \backslash \Gamma_{F}$. The inward normal vector $n$ at each point of the pore interface is oriented towards the fluid part of the sample, and is well-defined assuming there is sufficient regularity on $\Omega_{F}$. This condition is met provided the voxel sizes are sufficiently small compared to the pore dimensions.

In this article we focus on the reliable numerical simulation of microfluidic flows in three-dimensional porous rocks. For typical fluids and given the small dimensions of pore throats (micrometer scale or less), we are dealing with very low Reynolds number flows. In this flow regime, the dynamic momentum conservation law for the fluid phase, known as the Navier-Stokes equation, simplifies to the quasi-stationary Stokes equation. On the other hand, the fluid/solid interface featuring unresolved surface roughness can be elegantly modelled by a smooth interface with a slip boundary condition [20].

We therefore consider the three-dimensional incompressible Stokes equation, subjected to a downward pointing force field $f$, with a slip boundary condition at the interface $\Sigma$ :

$$
\begin{cases}-\mu \Delta u+\nabla p=f, & \text { in } \quad \Omega_{F} \\ \operatorname{div} u=0, & \text { in } \Omega_{F} \\ u-\beta T D(u) n=0, & \text { on } \Sigma \\ u \text { and } p \text { periodic } & \text { on } \Gamma_{F}\end{cases}
$$

with $u$ the fluid velocity, $p$ the pressure, $D(u)=\left(\nabla u+\nabla u^{T}\right) / 2$ the shear-rate tensor, $T=I-n \otimes n$ the projection operator on the tangential components, $\mu$ the dynamic viscosity assumed to be constant, and $\beta>0$ the slip coefficient. We impose three-dimensional periodic boundary conditions on $\partial \Omega$ since rock samples are typically constrained in a solid casing with a fluid layer on top and bottom when permeability tests are conducted (Figure 2). Provided the considered volume is sufficiently large to be representative, the lateral solid boundaries do not impact the results.

The boundary conditions at the pore interface $\Sigma$ are split according to the normal and tangential parts of the velocity and ensure, respectively, the impermeability of the interface and the slip along the tangential directions. In literature, two formulations can be found for the slip condition on the tangential velocity components: using either the velocity gradient whose formalism was detailed in [18] and [32], or the Navier condition involving the shear tensor, as considered in (3).

After segmentation of the $\mu \mathrm{CT}$ data, a binary dataset is obtained, with 1 for the solid phase and 0 for the liquid phase, that can mathematically be described by the function $\chi_{S}$. From this characteristic function of the solid phase $\chi_{S}$, the normal field $n$ oriented toward the fluid domain $\Omega_{F}$ has to be built in order to express the slip in equation (3).

In fact, the normal vector computation at the interface relies on the convolution principle with a Gaussian kernel chosen as

$$
\mathcal{G}_{\sigma}(X)=e^{-(X \cdot X) / 2 \sigma^{2}}
$$

with $(X \cdot X)$ the usual scalar product on $\mathbb{R}^{3}$ and a standard deviation $\sigma=h$ or $2 h$. An approximation of the inward unit normal $n$ (directed toward the fluid domain) is then given by

$$
n_{\mid \Sigma} \simeq-\frac{\nabla\left(\chi_{s} * \mathcal{G}_{\sigma}\right)}{\left\|\nabla\left(\chi_{s} * \mathcal{G}_{\sigma}\right)\right\|}
$$

with $\nabla\left(\chi_{s} * \mathcal{G}_{\sigma}\right)=\chi_{s} * \nabla \mathcal{G}_{\sigma}$. Assuming that $\Omega_{s}$ bounded, we then have $\chi_{s} \in \mathrm{L}^{1}\left(\mathbb{R}^{3}\right)$ and on the other hand, $\mathcal{G}_{\sigma} \in \mathcal{C}^{\infty}\left(\mathbb{R}^{3}\right)$ with all its partial derivatives of order one, which are bounded, to compute the gradient. Theoretically, $\chi_{s} * \nabla \mathcal{G}_{\sigma}$ is $\mathcal{C}^{1}\left(\mathbb{R}^{3}\right)$ and the normal vector is well-defined at each point of the interface $\Sigma$. We also introduce $\tau_{k}, k \in$ $\{1,2\}$ the tangential vectors to set the Robin boundary condition on the tangential velocity components. These vectors 


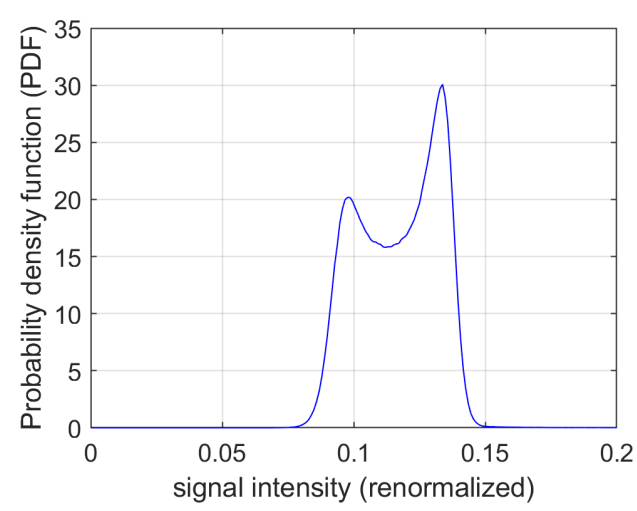

Fig. 3 Probability density function of the level of gray in the raw microCT image, providing a segmentation threshold between fluid and solid. The signal is normalized so that the image sensor values range from 0 to 1 for the maximum measurable intensity.

are computed such that $\left(n, \tau_{1}, \tau_{2}\right)$ is a direct orthonormal basis locally on the interface, meaning that if $n \times e_{k} \neq 0$ with $e_{k} \in\left(\overrightarrow{e_{x}}, \overrightarrow{e_{y}}, \overrightarrow{e_{z}}\right)$ a generator, we get:

$$
\tau_{1}=\frac{e_{k} \times n}{\left\|e_{k} \times n\right\|} \quad \text { and } \quad \tau_{2}=n \times \tau_{1}
$$

where $\left(\overrightarrow{e_{x}}, \overrightarrow{e_{y}}, \overrightarrow{e_{z}}\right)$ are the $\mathbb{R}^{3}$ usual canonical basis vectors. These normal and tangential fields are used to define the projector in the system (3) by

$$
T=I-n \otimes n
$$

which is equivalent to $T u=-n \times n \times u$ with $n$ variable in space along the fluid/solid interface.

It is noticeable that the slip condition $u-\beta T D(u) n=0$ in the system (3) expresses the tangential slip

$$
u \cdot \tau_{k}=\beta(D(u) n) \cdot \tau_{k}
$$

on the two tangential directions of the fluid/solid interface $\Sigma$ (for $k=1,2$ ), and at the same time one obtains the no-slip-through condition

$$
u \cdot n-\beta(T D(u) n) \cdot n=u \cdot n=0
$$

on the normal direction.

Moreover, we can consider a dimensionless formulation of (3) using the non-dimensional space variables $X^{*}=$ $X / L$ referred to as relative coordinates, where $X=(x, y, z)$ are the absolute coordinates and $L$ is the characteristic length, linked to the physical scale of the problem. The dimensionless Stokes problem is written $-\Delta u^{*}+\nabla p^{*}=f^{*}$ in $\Omega_{F}$ and $u^{*}-\beta^{*} T D\left(u^{*}\right) n=0$ on $\Sigma$ where $u^{*}=u / U$ depends on the characteristic velocity $U, p^{*}=L p / \mu U, f^{*}=$ $f L^{2} / \mu U$ and $\beta^{*}=\beta / L$. The other equations remain of the same type using star variables. The permeability tensor $K$ is determined from the pore-scale simulation by homogenization. In this study, we focus solely on the permeability estimate in the main flow direction so we end up with a scalar permeability defined by

$$
K=\phi \mu<u>_{\Omega_{F}} /<f>_{\Omega_{F}}
$$

where $<.>_{\Omega_{F}}$ represents the average in the fluid domain, $\phi$ the sample porosity and $\phi<u>_{\Omega_{F}}$ the so-called superficial velocity [33]. Equation (7) assumes that we can neglect the non-diagonal terms of the permeability tensor with respect to the diagonal terms. The porosity is numerically computed by taking $N_{F} / N_{V}$ with $N_{F}$ the number of grid points such that $\chi_{s}=0$ in the reference computational domain $\Omega$ and $N_{V}$ the total number of mesh points in the sample, excluding the casing. In this work the permeability estimation is obtained using the dimensionless formulation, yielding $K^{*}$, and the absolute permeability is then computed based on the dimensional relation $K=L^{2} K^{*}$. From this point forward on, we will work on the non-dimensional problem but the star notation will be omitted for the sake of readability.

\subsection{Test cases for model validation}

In section 4, our method that quantifies uncertainties is evaluated by means of four test cases, each having their own objectives. First, a perfect cylinder is studied. As an analytical solution exists for this particular test case, this offers a good opportunity for validation of the numerical method (section 4.1). Next, three real geometries are considered: a Bentheimer sandstone (section 4.2), a Castlegate sandstone (section 4.3) and a sand pack (section 4.4). All three datasets are obtained by X-ray tomography and are of comparable quality. Two of them were acquired at the DMEX Center for X-ray Imaging in France (Bentheimer, sand pack), while the other one (Castlegate) is freely available in the Digital Rocks data portal [34].

The primary objective of these test cases is to illustrate the model performance on a range of real samples featuring different morphological characteristics. The key difference between both sandstone samples is the average pore size, 
which is much larger for Bentheimer than for Castlegate. The sand pack has the advantage to present a pore space that is topologically very different from both sandstones, as well as a much higher porosity.

In addition to this primary objective, we also aim to consider whether the analysed sample volume must have a representative size. To that extent, the considered volume of the Bentheimer sandstone (a cube having $2^{8}$ cells of $2.2 \mu \mathrm{m}$ in each direction) was intentionally selected smaller than the REV (a factor two in each direction), while both other material geometries were representative: $2^{9}$ cells in each direction, of $5.6 \mu \mathrm{m}$ for Castlegate and $1.5 \mu \mathrm{m}$ for the sand pack.

$\mathrm{X}$-ray tomography is a technique that enables to non-destructively characterise the morphology of a sample in three-dimensions at the micron-scale. The technique relies on the material-specific attenuation of an x-ray beam when traversing a sample. By probing a sample from different angles and each time recording the intensity of the transferred beam, one can spatially reconstruct the regions with higher and lower attenuation. This result is represented under the form of a three dimensional tensor containing the local attenuation coefficients, whereby each tensor entry corresponds to a voxel, i.e. a three-dimensional pixel, having a given lateral dimension. At the DMEX center for X-ray imaging, we dispose of two tomographs, a Bruker Skyscan 1172 and a Zeiss Xradia Versa 510, respectively used to obtain the Bentheimer and the sandpack dataset.

The datasets used in this paper where filtered and segmented prior to be used as an input for the model. The filtration was done with an edge preserving $3 \times 3$ median filter and aims to reduce the image noise while avoiding to smooth the interfaces. The segmentation into phases was based on thresholding, where the threshold corresponds to the local minimum of the (normalised) histogram of the dataset, as illustrated for the sand pack example in Figure 3. More sophisticated image processing techniques certainly exist, but in the scope of this paper we favored a minimalist approach on data processing to demonstrate that this is sufficient for the proposed model.

\section{Linear deviation of permeability and slip dimensioning}

\subsection{Asymptotic expansion of the Stokes equation with slip}

The first approach to quantify the uncertainty on the permeability is (i) to estimate the permeability to no-slip flow $K_{0}$, and then (ii) to impose a small slip length $\beta$ (compared to the domain size) and estimate the resulting permeability $K_{\beta}$. $\left[K_{0}, K_{\beta}\right]$ can be used directly as a type of confidence interval if the value taken as the slip length is considered realistic. The linear deviation can be approximated as $L_{0} \simeq\left(K_{\beta}-K_{0}\right) / \beta$, provided that the slip length is sufficiently small in order to avoid the noise in the evaluation of $K_{\beta}$.

The aim of this section is to show that linear deviation can also be computed precisely in a straightforward fashion by solving a Stokes equation with non-homogeneous Dirichlet boundary conditions. To do so, we focus on the asymptotic development of the solution to the Stokes equation subject to a Navier boundary condition, with $\beta$ a small parameter compared to the image size, so as to obtain

$$
K_{\beta}=K_{0}+\beta L_{0}+\beta^{2} R(\beta)
$$

with a bounded remainder $R(\beta)$. This results in an estimation of the uncertainty interval $\left[K_{0}, K_{0}+\beta L_{0}\right]$ for the absolute permeability subject to a possible slip length $\beta$.

To simplify the readability of the remainder, we will set the problem in the fluid part $\Omega_{F}$ with the no-slip-through condition on its boundary $\Sigma$ and slip one on the tangential components which gives the following equations:

$$
\begin{aligned}
-\Delta u_{\beta}+\nabla p_{\beta}=f, & \text { in } \Omega_{F} \\
\operatorname{div} u_{\beta}=0, & \text { in } \Omega_{F} \\
T u_{\beta}-\beta T D\left(u_{\beta}\right) \cdot n=0, & \text { on } \Sigma \\
u_{\beta} \cdot n=0, & \text { on } \Sigma .
\end{aligned}
$$

The goal of asymptotic expansion is to provide a formal development of $u_{\beta}$ and $p_{\beta}$ with respect to the slip length $\beta$. So, we need to consider a second order asymptotic expansion given by

$$
u_{\beta}=U^{0}+\beta U^{1}+\beta^{2} r_{\beta}
$$

for the velocity and

$$
p_{\beta}=P^{0}+\beta P^{1}+\beta^{2} q_{\beta}
$$

for the pressure, where the profiles $U^{j}$ and $P^{j}$ are obtained from (10) and (11), $r_{\beta}, q_{\beta}$ designating the respective remainders. The profiles $U^{j}$ and $P^{j}$ are characterized by the result in appendix C: from equations (37), (41), (43), we can see that the functions $\left(U^{0}, P^{0}\right)$ satisfy the no-slip Stokes problem (45) written as follows

$$
\begin{cases}-\Delta U^{0}+\nabla P^{0}=f, & \text { in } \Omega_{F} \\ \operatorname{div} U^{0}=0, & \text { in } \Omega_{F} \\ U^{0}=0, & \text { on } \Sigma\end{cases}
$$




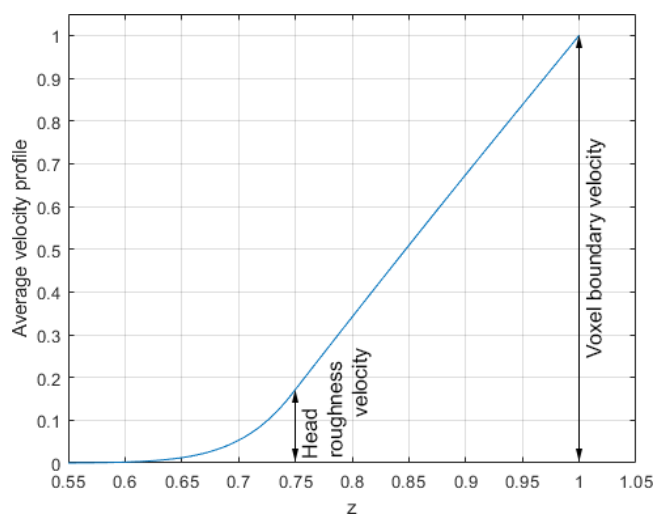

(a) Averaged velocity profile

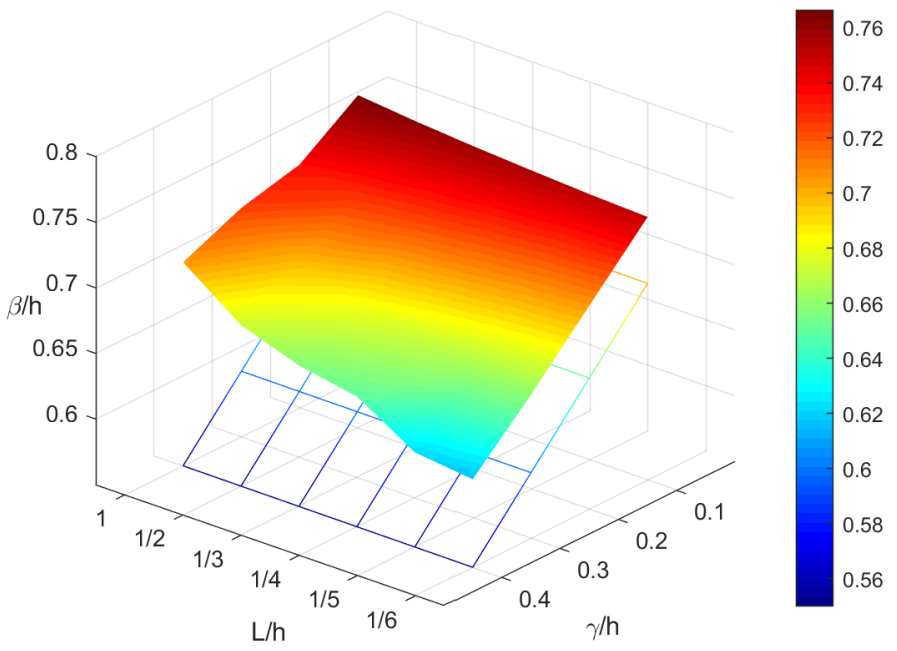

(b) Slip length induced by a $2 \mathrm{D}$ sinus roughness pattern

Fig. 4 To the left: Average velocity profile with respect to the z-axis for a roughness (13) with $\gamma / h=0.5, L / h=1 / 6$ and $\delta / h=0.5$ in non-dimensional coordinates. The slip coefficient is about $\beta / h=0.306$ in this case, when using a definition based on the box boundary velocity, and $\beta / h=0.046$ when based on the roughness head velocity. To the right: Evaluation of the slip coefficient with respect to the roughness amplitude and pitch for an X-ray absorption level of one quarter, in comparison to the flat case (no roughness). The slip coefficient estimation ranges from $\beta / h=0.617$ to $\beta / h=0.766$.

Furthermore, taking $k=1$ in equations (39), (41) and (44), developed in appendix C, we also obtain $\left(U^{1}, P^{1}\right)$ profiles that satisfy the following Stokes problem (46), which is written

$$
\begin{cases}-\Delta U^{1}+\nabla P^{1}=0, & \text { in } \Omega_{F} \\ \operatorname{div} U^{1}=0, & \text { in } \Omega_{F} \\ T U^{1}=T D\left(U^{0}\right) \cdot n, & \text { on } \Sigma \\ U^{1} \cdot n=0, & \text { on } \Sigma .\end{cases}
$$

Finally, we obtain the absolute permeability and its linear deviation by the first order Taylor development (8) of (7), i.e. $K_{0}=\phi \mu<U^{0}>_{\Omega_{F}} /<f>_{\Omega_{F}}$ and

$$
L_{0}=\phi \mu<U^{1}>_{\Omega_{F}} /<f>_{\Omega_{F}}
$$

where $U^{1}$ is the solution of (11) involving non-homogeneous Dirichlet boundary conditions, i.e. a prescribed slip velocity. Similarly, we easily obtain $R(\beta) \propto<r_{\beta}>\Omega_{F}$.

\subsection{Slip value and its link to multi-scale modeling}

In this section, we focus on estimating the values of the slip length which appropriately represent the uncertainties associated with the CT scan. In order to carry out possible slip length values and exhibit the bounds, we consider, in a computational box of size $h^{3}$, the periodic sinusoidal geometry at a mean height $\delta$ with an amplitude $\gamma$ and a pitch $L$ :

$$
\varphi(x, y)=\delta+\frac{\gamma}{2} \sin (2 \pi x / L) \sin (2 \pi y / L)
$$

defining the solid as the domain below the graph of this function (that is to say the point $(x, y, z)$ such that $z<\varphi(x, y)$ ), and the fluid domain above its graph.

The usual approach when modeling a roughness or its homogenization, is to set a Navier/Robin boundary condition that quantifies the slip at a given height relative to the top, mean line or bottom of the roughness pattern [32]. This leads to the relation (1) on the tangential velocity introduced previously:

$$
u-\beta D(u) n=0 \quad \text { on } \Gamma
$$

where $\Gamma$ is the smooth - or flat - reference surface on which a model of roughness effect is used or an homogenization of the roughness pattern (small scales of the surface $\Sigma$ ) is performed. In 1D, after averaging along the crosswise directions, the above equation is written $u(\zeta)-\beta u^{\prime}(\zeta)=0$ and we obtain Figure 4a: we can see that the reference height $\zeta$ (or equivalently the position of the surface $\Gamma$ ) can be chosen very closely to the effective surface (at the head of the roughness pattern, usually considered in nano-fluidics experiments describing this effective surface) or, in our case, at the edge of the voxel to be used as a meaningful boundary condition for the neighboring voxel. Despite a difference 


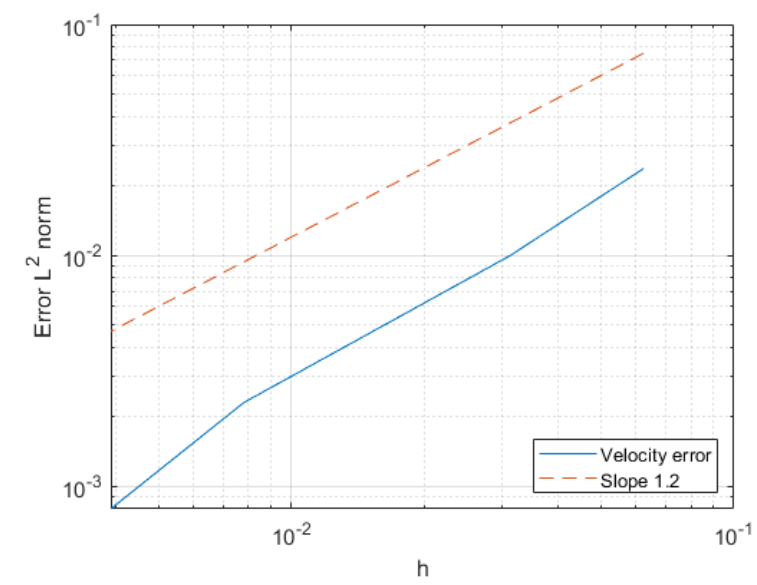

(a) Error on velocity

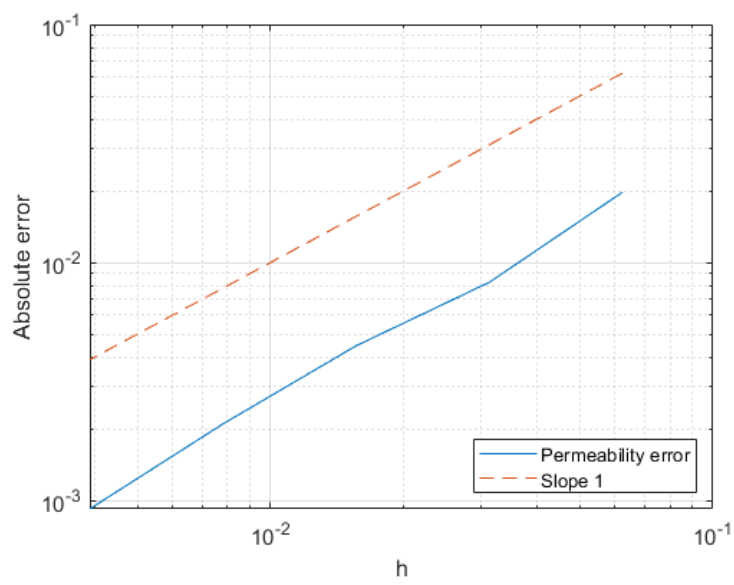

(b) Error on permeability

Fig. 5 Mesh convergence analysis in a cylindrical geometry for $\beta=0.1$ : (a) Velocity errorL ${ }^{2}$-norm with respect to the grid size for $2^{5}$ to $2^{9}$ cells in the lateral directions. (b) Absolute permeability error with respect to the grid size given the theoretical permeability (17).

in use between a slip at the roughness head, intrinsic to the surface, and a slip at the voxel edge, pertinent in image processing but linked to the voxel size, there is only one concept and definition of the slip condition and slip length.

However, whatever the choice of the reference velocity on this surface, as long as it is chosen above the solid, we can see in Figure 4a that the velocity gradient remains constant in the fluid part, which is quite a conventional result [16]. Consequently, the slip velocity based on the pixel size is proportional to the slip based on the head of the roughness pattern, obtained by microscopy or nano/micro-fluidics experiments. Such experiments can evaluate slip lengths depending on the typology of the roughness pattern. Moreover, according to different studies involving ridges or trenches [19, 22], pillars [24] and holes [25], or carbon nanotube coatings [23], for a given kind of pattern, the pitch and the solid fraction are the two most important parameters for determining slip lengths [26].

A direct consequence of the proportionality of the slip velocity is a shift in the slip length: an intrinsic slip length $\beta_{\text {exp }}$ at the top of the rough surface evaluated at $50 \%$ of the voxel width (at position $h / 2$ ), results in an effective slip at the voxel boundary equal to

$$
\beta \simeq \beta_{\text {exp }}+h / 2
$$

since $u(h)=\beta u^{\prime}(h)=u(h / 2)+h u^{\prime}(h / 2) / 2=\beta_{\exp } u^{\prime}(h / 2)+h u^{\prime}(h / 2) / 2 \simeq \beta u^{\prime}(h / 2)$ due to $u^{\prime}(h) \simeq u^{\prime}(h / 2)$ (e.g. see Figure 4a).

At a quantification level, Figure $4 \mathrm{~b}$ presents the slip lengths for a range of roughness amplitudes $0.1 \leq \gamma / h \leq 0.4$ and a range of pitches $1 / 6 \leq L / h \leq 1$. The mean solid position is set at $\delta / h=0.25$, corresponding to a "light gray" voxel due to its low absorption. Numerically, we used a GMRES method, detailed in appendix A, with a Krylov subspace size $m=20$, a given tolerance of $\varepsilon=10^{-4}$ and a grid resolution of $2^{6}$ cells in each direction, leading to a residual error of $5 \times 10^{-5}$. In such a situation, the maximum value tends to $\beta / h=0.75$, but in most cases the slip length belongs to the range $\beta / h \simeq 0.6-0.7$.

Beyond these estimations based on a case study of roughness, the hydrophobic and/or slip length reported in literature for multi-phase flows conventionally ranges from $200 \mathrm{~nm}$ [29] to $700 \mathrm{~nm}$ and even $950 \mathrm{~nm}$ [23]. For an mica/water interface with a roughness of $15 \mathrm{~nm}$, slip lengths in the range of $500-860 \mathrm{~nm}$ have been observed [13]. Moreover, Yao et al. [35] report roughness heights up to $400 \mathrm{~nm}$, for quartz sand, observed using optical interferometry. We note that Krinsley [36] reports different roughness patterns for quartz grains, depending on its depositional environment (aqueous, high energy beach, desert, hill, fjord). The resolution $h$ of the samples considered in the present study range from $1.5 \mu \mathrm{m}$ for the sandpack and $2.2 \mu \mathrm{m}$ for the Bentheimer sandstone to $5.6 \mu \mathrm{m}$ for the Castlegate sandstone. For fine resolutions, choosing a slip length of $\beta / h$ in the range $0.5-0.8$ is then coherent for both the under-resolved roughness and the blur of fluid/solid interface, especially when the blur is limited to 1 voxel. Of course it is possible to consider larger slip length to quantify the uncertainty induced by wider blurred layers. To conclude, in the following sections, we consider slip lengths at $\beta / h=0.5$ and 0.76 .

\section{Absolute permeability deviation analysis on real geometries of porous rocks}

\subsection{Flow in a cylindrical domain}

We first perform convergence analysis by studying the Stokes flow in a vertical cylinder of radius $R$ with a slip boundary condition along the lateral boundary. The goal is to validate the numerical method presented in this work and detailed in 


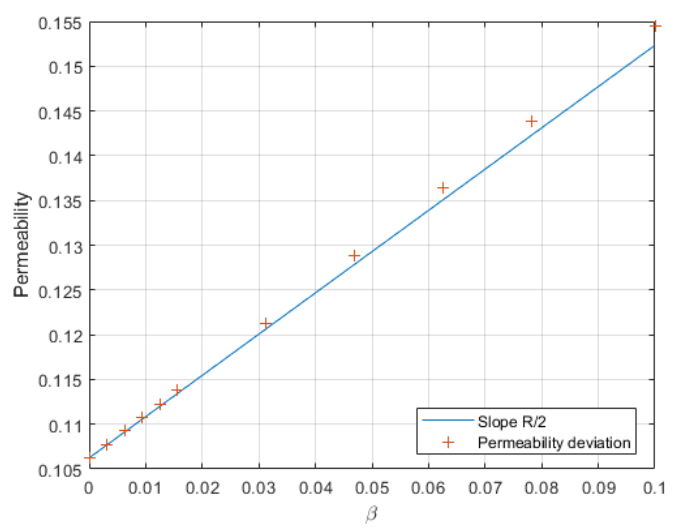

Fig. 6 Permeability deviation in relative coordinates (nondimensional) with respect to $\beta$ on a grid of $2^{6}$ cells in each direction and using the permeability with adherent pore interface, denoted $K_{0}$, as a reference. A slope of $R / 2$ is expected for the permeability deviation.

appendix A, and at the same time to exhibit the features of a pore with a simple geometry, whose flow has an analytical solution.

The exact solution, dependent on $\beta$ is given, in cylindrical coordinates, by

$$
u(r, \theta, z)=\frac{\left(R^{2}-r^{2}\right)}{4 \mu} f+\beta \frac{f R}{2 \mu} .
$$

with $f=-\overrightarrow{e_{z}}$. The analytical expression for the dimensionless permeability as a function of $\beta$ reads:

$$
K_{\beta}=\frac{R^{2}}{8}+\beta \frac{R}{2}
$$

In this case, the linear deviation is exact and the development of $K_{\beta}$ contains no remainder after the first order term. The total permeability deviation $K_{\beta}$ corresponds to $K_{0}+\beta L_{0}$, with $L_{0}=R / 2$, and hence provides a single confidence interval.

The analytical permeability is computed by taking the cylinder as the whole porous domain, meaning we assume here that $\phi=1$. The computational box is the unit box $[-1,1]^{3}$ and the cylindrical geometry on the grid is defined by all the grid points whose distance from the vertical axis is smaller than 0.9 , which statistically corresponds to a cylinder of radius $\mathrm{R}=0.922$ (the solid being strictly outside, this gives an effective radius larger than 0.9 ). In the present work, we are dealing with two different diagnostics: on the one hand, with $\beta=0.1$, mesh convergence curves for the velocity error $\mathrm{L}^{2}$-norm and the permeability estimate are presented. On the other hand, we monitor the permeability deviation with respect to $\beta$ for a given mesh resolution. The convergence diagnostics in Figure 5 are performed with a Krylov space size set to $m=50$, a given tolerance of $\varepsilon=10^{-6}$ and up to a resolution of $2^{9}$ cells, except in the main flow direction where we set $2^{5}$ cells. In fact, since both the analytic and numerical solutions are invariant with respect to the vertical axis, we consider only a thin layer for the validation study (without loss of generality) and thereby we limit the memory requirements to below $8 \mathrm{~GB}$ of RAM.

We observe a convergence of the order of $\mathcal{O}\left(h^{1.2}\right)$ for the velocity, which is slightly weaker than in the case of an adherent pore interface, since slip boundary conditions tend to disrupt the GMRES convergence. Moreover, this convergence order is lower than the order two finite difference schemes used in the numerical method, mainly because of the cramming effect on this cylindrical case. Concerning the permeability estimate, we get an order one convergence with respect to the mesh resolution with an error on the permeability of $1.9 \%$ for the coarsest resolution grid and $0.09 \%$ for the finest one. We made sure that the permeability deviation due to the slip boundary condition is not encompassed in the numerical error. When we represent the permeability evolution as a function of $\beta$, with the slip coefficient taking values between 0 and 0.1 , we recover the theoretical slope of $L_{0}=R / 2$ confirming the precision of the derivation and implementation (Figure 6).

Another meaningful investigation that was performed on this validation case is the monitoring of the boundary condition at the approximate interface: it is satisfied with an error of $10^{-3}$ even for the coarsest considered grid $\left(2^{5}\right)$ which means that the slip boundary condition is properly controlled with the proposed method. This observation highlights the ability of this direct approach to deal with slip flows.

\subsection{Flow inside a Bentheimer sample}

This section is dedicated to an initial application on a real porous rock: the Bentheimer sandstone sampled at a resolution of $256^{3}$, corresponding to a physical size of $L=0.56 \mathrm{~mm}$ with $2.2 \mu \mathrm{m}$ wide voxels.

We are first interested in analysing the memory storage requirements in order to identify the Krylov subspace size we can afford in practice, using the numerical method detailed in appendix A. The memory cost mainly depends both on the grid resolution and the Krylov subspace size, so we will present it as a function of the total number of mesh 


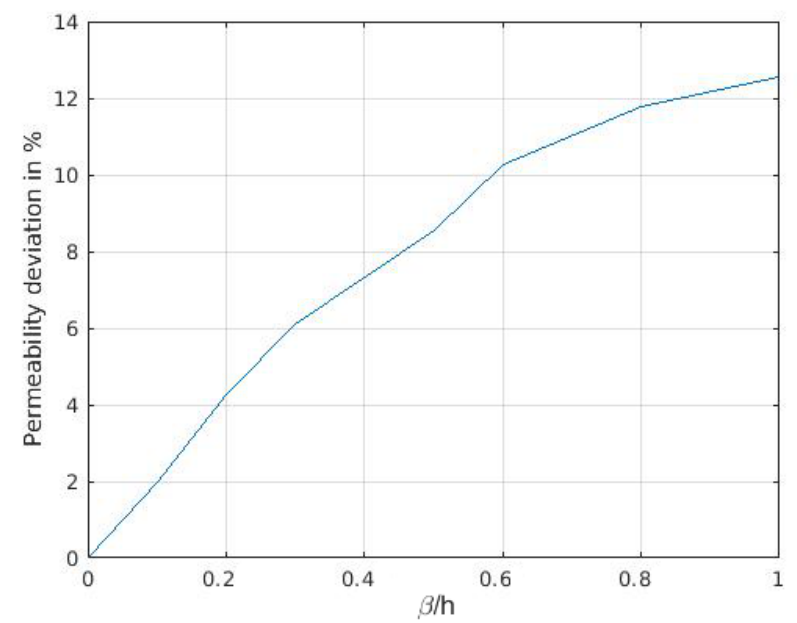

Fig. 7 Permeability deviation, on the Bentheimer geometry, computed by (7) with respect to the slip coefficients: for $\beta / h=0.5$, the permeability deviation is about $8.56 \%$.
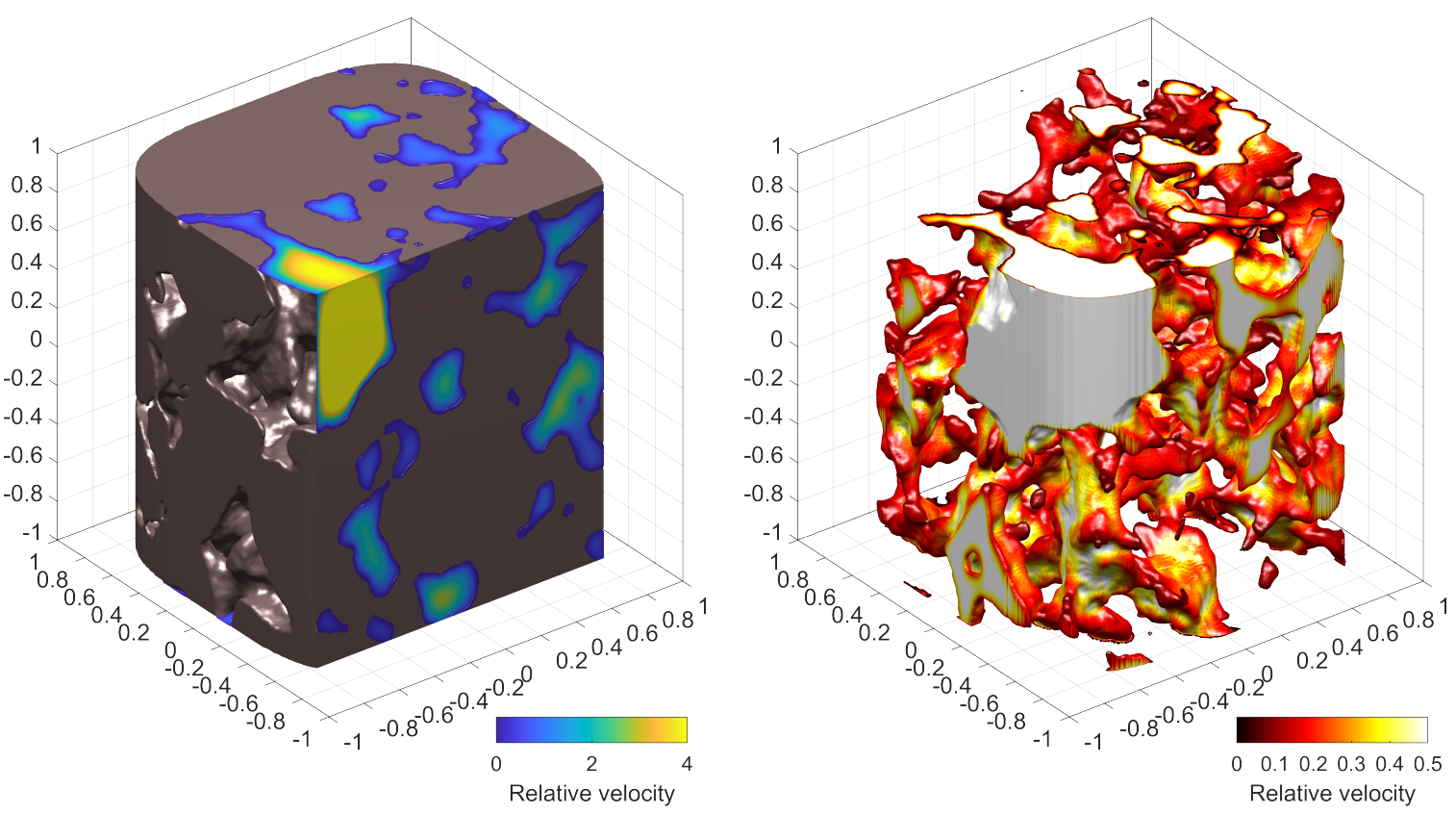

Fig. 8 Bentheimer geometry with a slip coefficient of $\beta / h=0.5$ : Relative velocities in the fluid domain (to the left) and at the fluid/solid interface (to the right). Resolution of $2^{8}$ cells per direction. The gray part represents the solid matrix constrained in an impermeable solid casing.

points $N$ and the Krylov size $m$. Five tables, mainly, are used to call the Fortran GMRES(m) subroutine: $\mathrm{X}$ to store the unknowns, $\mathrm{Y}$ for the result of the matrix-vector product function, $\mathrm{B}$ for the right-hand-side, all of size $4 N$, and additionally a vector $V$ of size $4 N(m+1)$ to store the orthonormal basis $\left\{v_{1}, \ldots, v_{k+1}\right\}$ of the Krylov subspace obtained by Arnoldi's process and finally one table of size $N$ to characterize each mesh point with respect to its solid, fluid or first layer of identified solid. Then, the GMRES algorithm itself requires the storage of the Hessenberg matrix of size $(m+1) m$ (defined in appendix A) and four additional tables (three of size $m$ and one of size $m+1$ ) for solving the least squares problem (23). This leads to a total storage space of $14 N+4 N(m+1)+(m+1)^{2}+3 m$.

The simulations are performed using 16GB of RAM, such that the Krylov space is set to $m=20$. However, it must be noted that the Krylov subspace size has an effect on the convergence rate. This highlights the difficulty in choosing an appropriate value of $m$, especially when the system size grows. Therefore, in the next section we propose a strategy for improving convergence in high resolution simulations.

As the physical parameters, the dynamic viscosity is set to $\mu=10^{-3} \mathrm{Nm}^{-2} s$ and the driving force to $f=$ $5000 \mathrm{Nm}^{-3}$. To study the impact of the under-resolved roughness on the permeability, the slip coefficient range is taken between 0 and $h$ with a particular interest in $\beta / h=0.5$ which helps provide an upper bound for the permeability deviation when the X-ray absorption level is estimated to one half. The permeability deviation with respect to the slip coefficients are presented in Figure 6. 

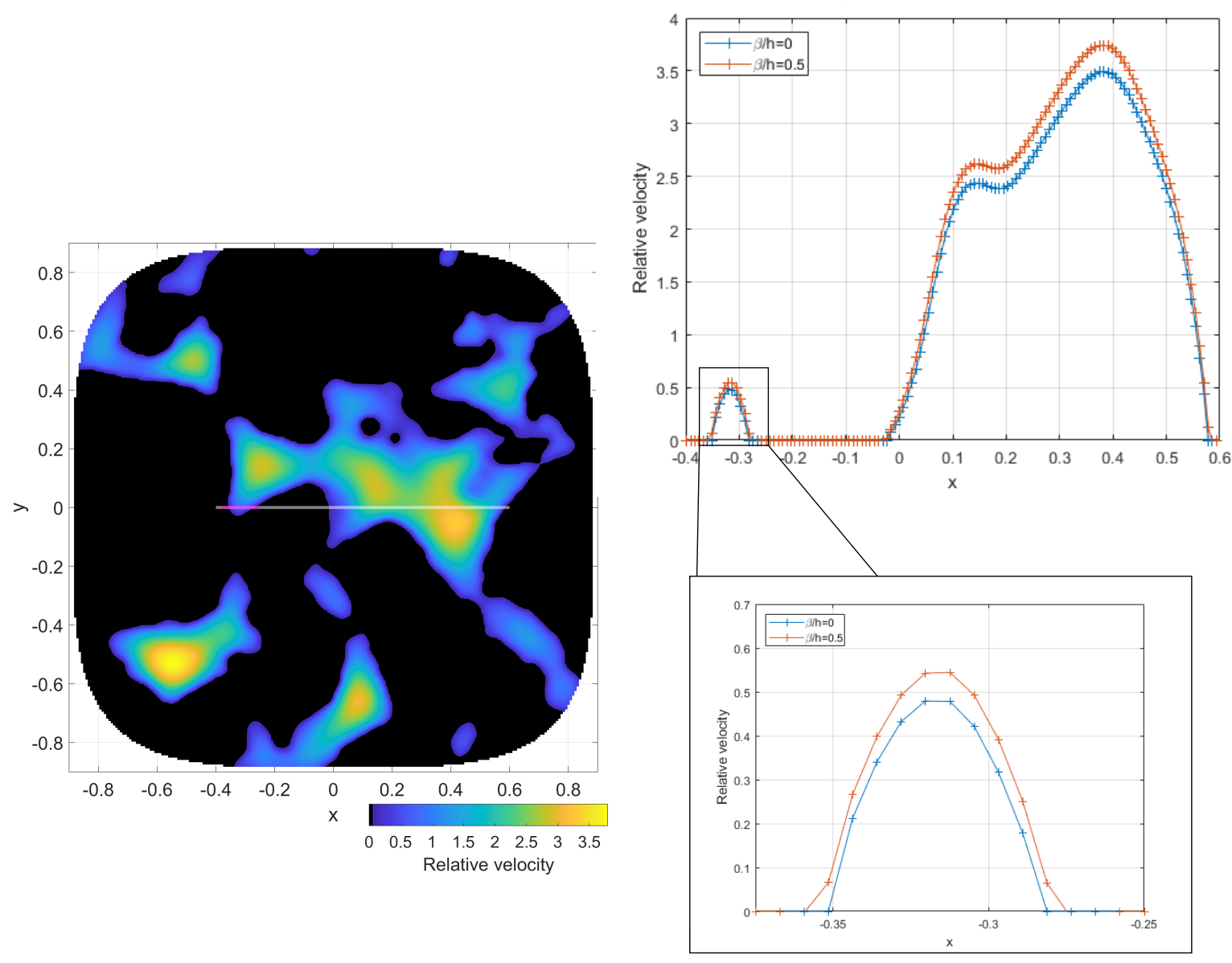

Fig. 9 Relative velocities in the main flow direction and on a sectional profile (taken at $y=0$ and $z=-0.5$ in relative coordinates) on the Bentheimer geometry: comparison between the adherent and slip boundary conditions for $\beta / h=0.5$. The $z=-0.5$ slice for a slip length of $\beta / h=0.5$ is displayed on the left image, the white and magenta lines respectively show the cut for the unzoomed and zoomed curves to the right.

The permeability with an adherent pore interface, denoted $K_{0}$, is taken as reference for the computation of the relative deviation of absolute permeability, given by

$$
\frac{\left|K_{\beta}-K_{0}\right|}{K_{0}}
$$

where $K_{\beta}$ is the permeability with slip boundary condition on the pore interface. In the adherent case, after 100 iterations we obtained a dimensional permeability of $2.87 \times 10^{-11} \mathrm{~m}^{2}$, noticing that the usual values obtained from experimental measurements are around $10^{-12} \mathrm{~m}^{2}$, and this actual Bentheimer sample at a representative scale has been measured at 2-3 $\mu^{2}$ [37]. The difference is not necessarily significant, as the numerical porosity of the sample considered is about $\phi=28.75 \%$, while typical values for the Bentheimer sandstone are rather around $24 \%$ [38]. Finally, the pore interface represents about $7.18 \%$ of the sample geometry, excluding the solid casing. In order to provide a physical characterization of the pore interface, we introduce the specific area of the porous medium defined as the ratio of the pore interfacial surface area per unit volume, expressed in $m^{-1}$. This quantity is numerically estimated and provides a specific area of about $A_{s}=25027 m^{-1}$.

The relative residual norms quantifying the convergence of the numerical method, and the permeability changes with respect to the iterations are displayed in Figures $15 \mathrm{a}$ and $15 \mathrm{~b}$, on which we compare $\beta / h=0$ for $\beta / h=$ 0.5 . For the latter, the relative residual norm and the permeability estimation scale respectively around $10^{-2}$ and $3.11 \times 10^{-11} \mathrm{~m}^{2}$ which represents a permeability deviation of $8.56 \%$ for such a slip coefficient. This deviation increases to about $12 \%$, as illustrated in Figure 7 by the full permeability deviation analysis performed with slip coefficients between 0 and $h$. Both the 3D sample geometry and the final relative velocity field with slip pore interface, in the $\beta / h=0.5$ case, are shown in Figure 8 .

This example highlights that even a small slip coefficient induces a significant permeability deviation since the pore boundary condition induced by an invisible roughness leads to a different evaluation of the flow rate, as illustrated in Figure 9. Actually, we present here a sectional profile of the relative velocities in the main flow direction for adherent and slip boundary conditions for $\beta / h=0.5$. The related 2D pore slice and the exact $1 \mathrm{D}$ segments for the main profile and its zoomed version are also displayed on the left. It remains that one should carefully consider this uncertainty on 


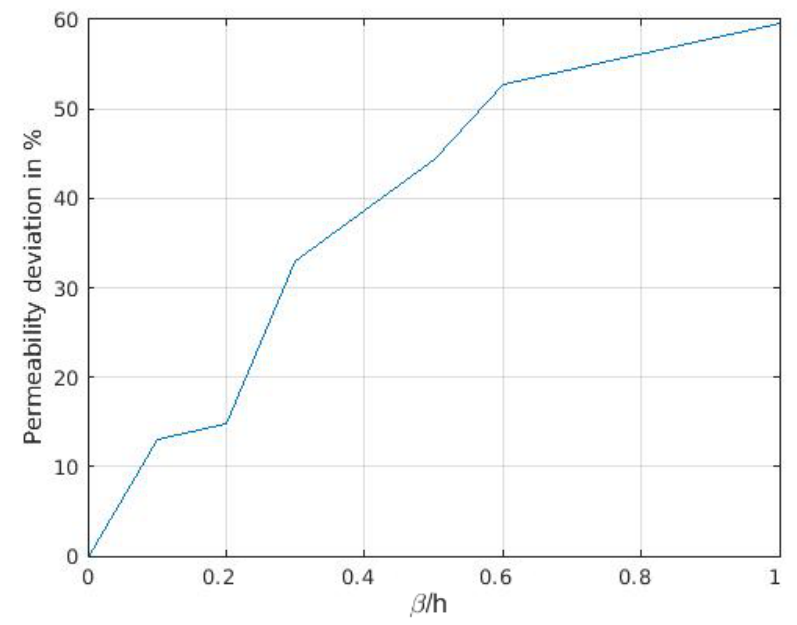

Fig. 10 Permeability deviation with respect to the slip coefficients on a Castlegate sandstone sample for a $2^{8}$ resolution per direction. The reference permeability $K_{0}$ is given by $2.09 \times$ $10^{-11} \mathrm{~m}^{2}$ in this case.

the permeability estimate to provide, instead, a permeability range thanks to tomographic dataset, especially when the absorption level does not enable us to precisely grasp the pore interface roughness.

\subsection{High resolution flow inside a Castlegate sample}

The second real geometry considered in this study is a highly porous Castlegate rock whose numerical porosity is about $\phi=25.10 \%$. This sandstone outcrop was sampled in southeastern Utah, USA [34]. The numerical sample is extracted from the experimental X-ray dataset using a physical voxel size of $5.6 \mu \mathrm{m}$ and has a physical size of $L=2.8 \mathrm{~mm}$. This Castlegate sandstone is segmented on a $512^{3}$ grid, between fluid and solid points: the pore interface, characterized by the grid points among the solid ones but in contact to fluid, denoted $\Sigma_{s}$, represents about $12.63 \%$ of the sample geometry without a casing. Finally, in this sample, the specific area is numerically estimated and we get about $A_{s}=15678 \mathrm{~m}^{-1}$.

In such high resolution simulations, the Krylov subspace size is clearly limited regarding memory storage and consequently, the convergence slows down in comparison with a lower resolution grid. We thus use preconditioning, developed in appendix B, to obtain convergence results that are reasonable for well-resolved real geometries. The process consists in computing the velocity and pressure fields on a coarser grid, with a Krylov size that gives good convergence results for the residual norm: around $10^{-4}$ after 100 iterations for a 56GB RAM simulation with a Krylov size of $m=100$, which speeds up dramatically the global simulation.

For the physical parameters, we take $\mu=10^{-3} \mathrm{Nm}^{-2} s$ for the dynamic viscosity and $f=2000 \mathrm{Nm}^{-3}$ for the driving force. The full permeability deviation analysis, displayed in Figure 10, with a slip coefficient between 0 and $h$, is performed with the $2^{8}$ resolution per direction. For high resolution simulations, we only consider two main slip coefficient values: $\beta / h=0.5$ and $\beta / h=0.76$ previously justified. The reference permeability on the adherent pore interface, obtained using the preconditioning process detailed before, is about $K_{0}=1.59 \times 10^{-11} \mathrm{~m}^{2}$ after 200 iterations. The Krylov subspace size is set to $m=50$ on the thinner grid such that this permeability estimate is provided with a relative residual norm of less than $10^{-6}$. In this case, both the evolution of the relative residual norms and the permeability changes with respect to the number of iterations are presented in Figures $15 \mathrm{c}$ and $15 \mathrm{~d}$ respectively, on which we compare the adherent case with slip pore boundary by taking $\beta / h=0.5$. Figure 11 also shows the final relative velocity field at the full resolution, obtained in a slip pore boundary case.

By considering a slip coefficient of $\beta / h=0.5$, after the preconditioning process we reach a final relative residual norm of around $10^{-5}$, which is larger than for the adherent case. This can be explained by the fact that slip boundary conditions tend to disrupt the convergence since the slip often leads to a lack of coercivity in the variational formulation. The permeability evolution scales at about $2.57 \times 10^{-11} \mathrm{~m}^{2}$ which represents a permeability deviation of $61.63 \%$ compared to the adherent case. Finally, a slip coefficient of $\beta / h=0.76$, corresponding to light gray voxels, provides a permeability of $3.05 \times 10^{-11}$ with a final relative residual norm of $10^{-5}$. It represents a permeability deviation of $91.82 \%$ which means that $K_{\beta}$ is nearly twice the reference permeability $K_{0}$ for such a slip coefficient. We note that we get substantially higher permeability deviations on the Castlegate sandstone sample compared to the Bentheimer sample. This can be explained by a higher proportional pore interface (the pore interface represents $7.18 \%$ on the Bentheimer and $12.63 \%$ on the Castlegate). In fact, since the Castlegate geometry presents a large variety of small pores, the slip boundary conditions on the pore interface more significantly impact the velocity and thereby the permeability.In that regard, Figure 12 shows the relative velocities, for $\beta / h=0$ and 0.5 , on a sectional profile as well as the related $2 \mathrm{D}$ pore slice and the exact $1 \mathrm{D}$ segment where the graph and its zoomed version are taken. 


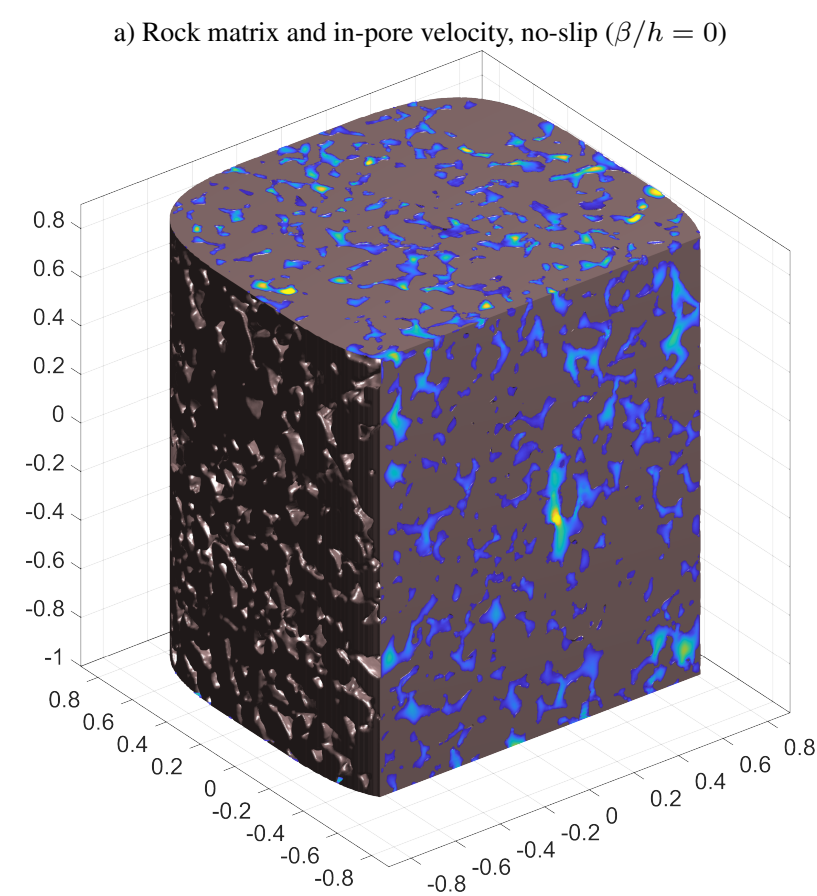

b) Rock matrix and in-pore velocity, slip length $\beta / h=0.76$

c) Interpolated slip at interface, slip length $\beta / h=0.76$
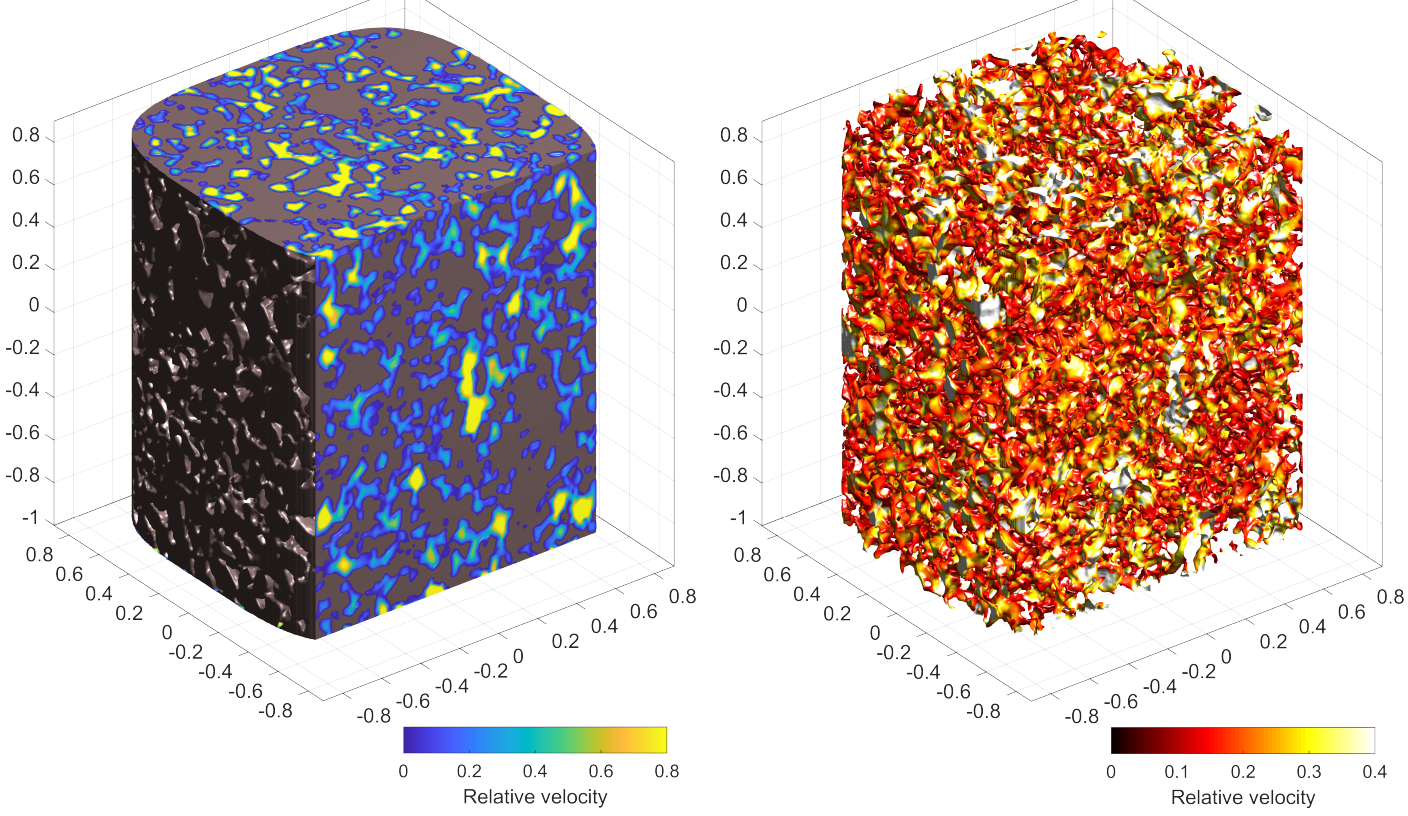

Fig. 11 Flow in the Castlegate geometry $\left(512^{3}\right)$ with adherent boundary condition (top image), and with slip coefficient $\beta / h=0.76$ ("lightgray" case in the bottom left image). Bottom right image: interpolated slip at fluid/solid interface. Flow pictures are colored by the norm of relative velocity with the same scale $(0-0.8)$, with respect to relative coordinates (from -1 to 1 ).

\subsection{High resolution flow inside a sand-pack sample}

The numerical study of the sand pack considered in this last case involve a grid resolution of $2^{9}$ cells in each direction so that the numerical sample extracted from the X-ray dataset has a physical length of $L=0.768 \mathrm{~mm}$ with $1.5 \mu \mathrm{m}$ wide voxels. Porosity and specific area are computed, respectively, at $\phi=45.43 \%$ and $A_{s}=93174 m^{-1}$. In this case, the pore interface represents about $20.04 \%$ of the grid points that model the sample geometry.

As described before, preconditioning starts on a twice coarser grid with a Krylov space size of $m=100$, followed by $M_{4}^{\prime}$ interpolation and computations on the more refined grid with $m=50$. The physical parameters involving the dynamic viscosity and the driving force are taken as described in the previous section.

The reference permeability on the adherent pore interface is about $K_{0}=1.02 \times 10^{-11} \mathrm{~m}^{2}$ after 200 iterations, obtained with a relative residual norm around $10^{-6}$. In Figures $15 \mathrm{e}$ and $15 \mathrm{f}$ we compare both the evolution along the preconditioning process of the relative residual norm and the permeability for $\beta / h=0,0.5$ and 0.76 . The permeability reaches $2.48 \times 10^{-11}$ and $2.98 \times 10^{-11} \mathrm{~m}^{2}$, respectively, for these two slip coefficient values. This means that on such 

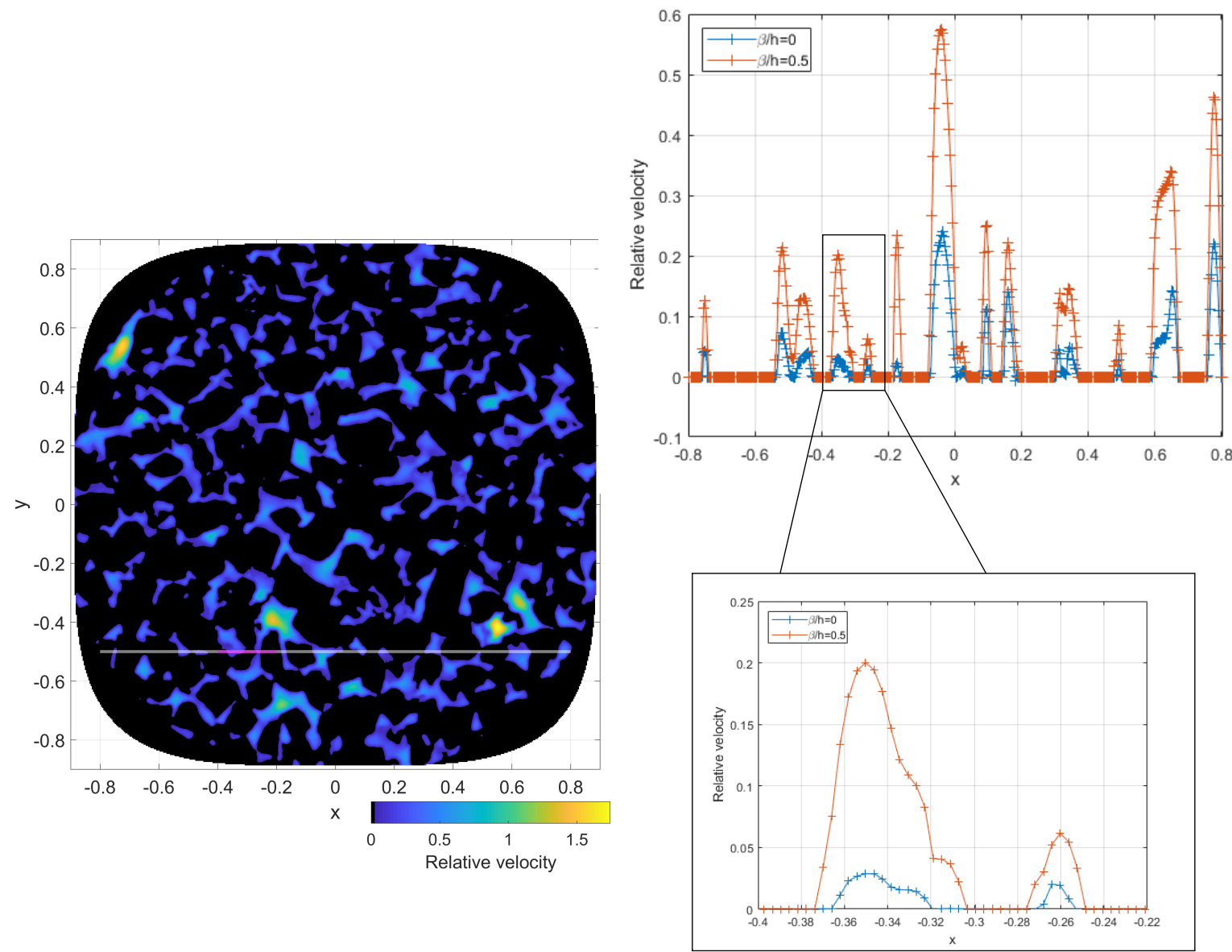

Fig. 12 Relative velocities in the main flow direction and on a sectional profile (taken as $z=0$ and $y=-0.5$ in relative coordinates) on the Castlegate geometry: comparison between adherent and slip boundary conditions for $\beta / h=0.5$. The slice $z=0$ for a slip length $\beta / h=0.5$ is displayed on the left-hand image, the white and magenta lines show the respective locations of the cuts of the unzoomed and zoomed curves to the right.

a) Rock matrix and poral velocity, no-slip case $(\beta=0)$

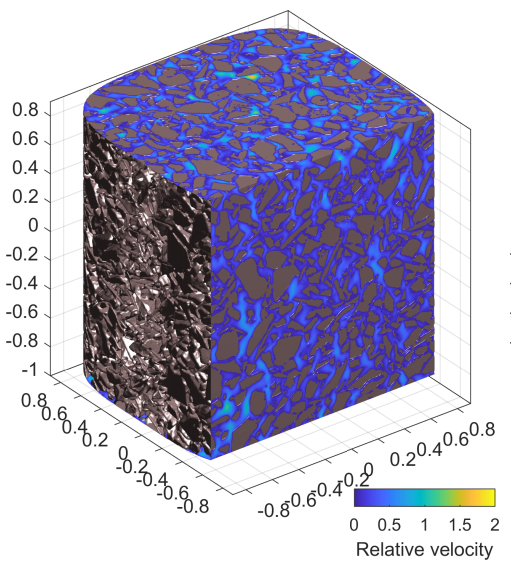

b) Rock matrix and poral velocity, slip length $\beta / h=0.76$

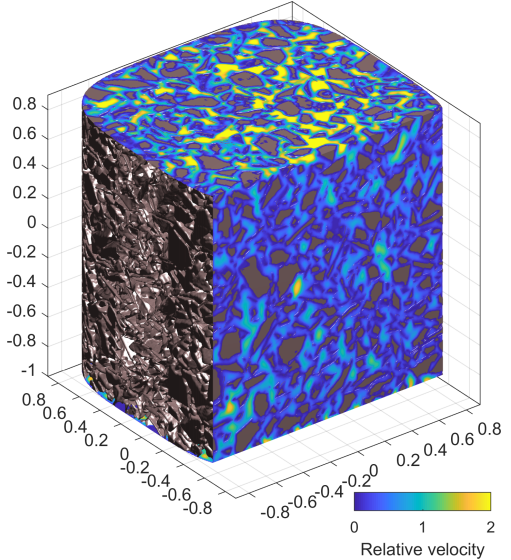

c) Interpolated slip at interface, slip length $\beta / h=0.76$

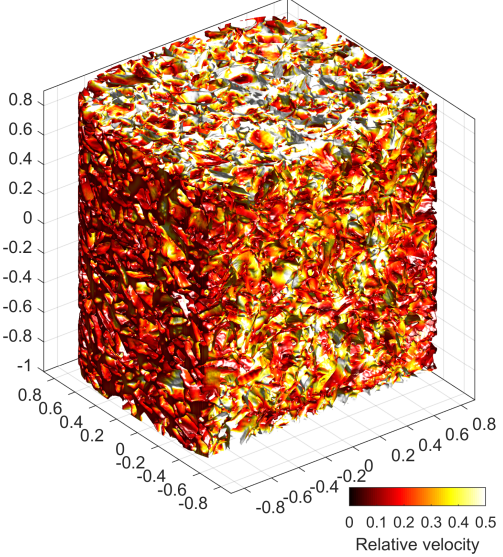

Fig. 13 Flow in the compact sand pack geometry $\left(512^{3}\right)$ with adherent boundary condition (left picture), with slip $\beta / h=0.76$ (middle picture), and slip at pore boundaries (right picture). Same color legend as for Figure 11. 

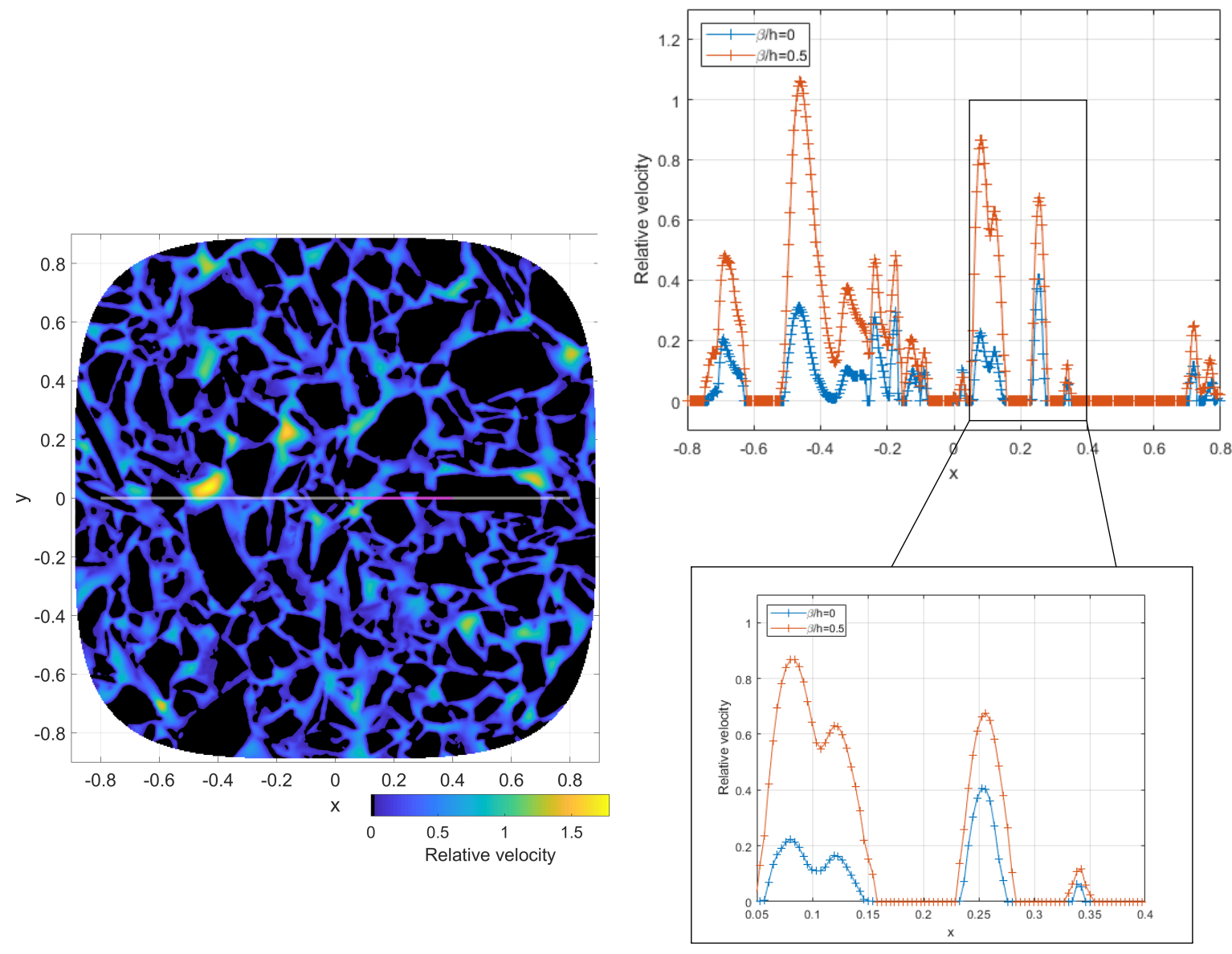

Fig. 14 Relative velocities in the main flow direction and through a sectional profile (taken at $z=-0.75$ and $y=0$ in relative coordinates) on the sand geometry: comparison between the adherent and slip boundary conditions for $\beta / h=0.5$. The slice $z=-0.75$ for a slip length $\beta / h=0.5$ is displayed on the left-hand image, the white and magenta lines show the location,respectively, of the cuts of the unzoomed and zoomed curves to the right.

geometries the permeability deviations are even greater than in the previous applications and scale at about $143 \%$ and $192 \%$.

As for the previous samples, Figure 13 shows the 3D geometry at full resolution with the final velocity fields obtained in the adherent and a slip pore boundary cases. Moreover, a 2D pore slice (taken in the main flow direction) is also illustrated on the left-hand side of Figure 14 to characterize the sectional profiles presented on the right. It shows the impact of the slip boundary condition (in this case $\beta / h=0.5$ ) on the flow rate.

Furthermore, we verify in this case that the permeability variation does not reflect the residual errors of the numerical scheme: the bottom images of Figure 15, show that the relative residual is the same for the two slip lengths $\beta / h=0.5$ and 0.76 .

\subsection{Results summary}

Table 1 summarizes the results obtained for both the raw deviations and the linear deviations for all considered samples. The permeability ranges account for the sample resolution, its bulk permeability and its geometry at pore scale.

We observe that the permeability deviations using the raw values (the full deviation) and the values based on the linear momentum are consistent, despite a small non-linear effect (as expected and shown in Figure 6).

Furthermore, for the different samples, a dimensionless ratio is computed from the relative deviation and the specific area which have the same units $m^{-1}$. This ratio is displayed in table 2 . It is theoretically equal to 2 for a cylindrical pore. It appears that the Bentheimer sandstone, of size $256^{3}$ and too coarse to be a representative sample, has a ratio $L_{0} / A_{s} K_{0}$ which is close to that of the cylindrical case.

Oppositely, the samples representative of their media, namely the Castlegate sandstone and the sand pack, have ratios in the range 12-17. We note that their porosity values are quite different. Future work will investigate whether or not sandstones with porosities in the range of $20-25 \%$ correlate with a universal dimensionless ratio (around 12). 


\begin{tabular}{|c|c|c|c|c|c|c|}
\hline \multirow[b]{2}{*}{ Sample } & \multirow[b]{2}{*}{$\begin{array}{c}\text { Slip-length } \\
\beta\end{array}$} & \multirow{2}{*}{$\begin{array}{c}\text { No-slip } \\
\text { permeability } \\
K_{0}\end{array}$} & \multicolumn{2}{|c|}{ Full deviation } & \multicolumn{2}{|c|}{ Linear deviation } \\
\hline & & & $\begin{array}{c}\text { Relative deviation } \\
\qquad K^{\prime} / K_{0}\end{array}$ & $\begin{array}{c}\text { Range } \\
{\left[K_{0}, K_{\beta}\right]}\end{array}$ & $\begin{array}{c}\text { Relative deviation } \\
L_{0} / K_{0}\end{array}$ & $\begin{array}{c}\text { Range } \\
{\left[K_{0}, K_{0}+\beta L_{0}\right]}\end{array}$ \\
\hline Unit & $\mu m$ & $\mu m^{2}$ & $m^{-1}$ & $\mu m^{2}$ & $m^{-1}$ & $\mu m^{2}$ \\
\hline Cylinder & - & $R^{2} / 8$ & $4 / R$ & {$\left[K_{0}, K_{0}+\beta R / 2\right]$} & $4 / R$ & {$\left[K_{0}, K_{0}+\beta R / 2\right]$} \\
\hline Bentheimer & 1.1 & 28.7 & 77818 & {$[28.7,31.1]$} & 63727 & {$[28.7,30.7]$} \\
\hline Castlegate & 4.26 & 15.9 & 215540 & {$[15.9,30.5]$} & 185956 & {$[15.9,28.5]$} \\
\hline Sandpack & 1.14 & 10.2 & 1684211 & {$[10.2,29.8]$} & 1561404 & {$[10.2,28.4]$} \\
\hline
\end{tabular}

Table 1 Summary of absolute permeability ranges obtained for the three samples and the ideal cylindrical pore, in both the full deviation and the linear deviation models. We use the notation $K^{\prime}=\left(K_{\beta}-K_{0}\right) / \beta$.

\begin{tabular}{|c|c|c|c|c|c|c|}
\hline Sample & $\begin{array}{c}\text { Computed } \\
\text { porosity } \\
\phi\end{array}$ & $\begin{array}{c}\text { Specific } \\
\text { area } \\
A_{s}\end{array}$ & $\begin{array}{c}\text { Relative } \\
\text { deviation } \\
K^{\prime} / K_{0}\end{array}$ & $\begin{array}{c}\text { Ratio } \\
K^{\prime} / K_{0} A_{s}\end{array}$ & $\begin{array}{c}\text { Relative } \\
\text { linear deviation } \\
L_{0} / K_{0}\end{array}$ & $\begin{array}{c}\text { Ratio } \\
L_{0} / K_{0} A_{s}\end{array}$ \\
\hline Unit & - & $m^{-1}$ & $m m^{-1}$ & - & $m m^{-1}$ & - \\
\hline Cylinder & $100 \%$ & $2 / R$ & $4 / R$ & 2 & $4 / R$ & 2 \\
Bentheimer & $28.75 \%$ & 25 & 78 & 3.1 & 63.7 & 2.5 \\
Castlegate & $25.1 \%$ & 15.7 & 215.5 & 13.7 & 186 & 11.9 \\
Sandpack & $45.4 \%$ & 93 & 1684 & 18.1 & 1561 & 16.8 \\
\hline
\end{tabular}

Table 2 Comparison of relative deviations (rounded) with respect to specific areas for the different samples, using the values of $K^{\prime}$ from table 1 .

\begin{tabular}{|c|c|c|c|c|c|c|}
\hline Sample & $\begin{array}{c}\text { Computed } \\
\text { porosity } \phi\end{array}$ & $\begin{array}{c}\text { Grain } \\
\text { size }\end{array}$ & $\begin{array}{c}\text { Charact. } \\
\text { length } \sqrt{K_{0}}\end{array}$ & $\begin{array}{c}\text { Sample } \\
\text { width }\end{array}$ & $\begin{array}{c}\text { Sample } \\
\text { resolution }\end{array}$ & $\begin{array}{c}\text { Sample } \\
\text { volume }\end{array}$ \\
\hline Unit & - & $\mu m$ & $m m$ & $\mu m$ & - & $m^{3}$ \\
\hline Bentheimer & $28.75 \%$ & $200-330$ & 5.3 & 563 & $256^{3}$ & 0.131 \\
Castlegate & $25.1 \%$ & $\simeq 150$ & 4.0 & 2867 & $512^{3}$ & 17.30 \\
Sandpack & $45.4 \%$ & $\simeq 50$ & 3.2 & 768 & $512^{3}$ & 0.332 \\
\hline
\end{tabular}

Table 3 Rock and numerical sample features. Orders of magnitude of grain size are taken from [39, 37] and [40]. The sample volume excludes the digital cell surrounding the rock matrix.

In any case, this tends to show that providing values of permeability by direct numerical simulation is hazardous if the specific area is not correctly estimated. When the interface area is properly computed, the interval of uncertainty becomes reliable as a range of permeability values.

\section{Conclusion}

Numerical modeling of flow in porous media at pore scale can be based on datasets obtained by X-ray micro-tomography. However, the finite resolution of these tomographic scans leads to an uncertainty on the position and morphology of the pore walls, which in turn impacts the effective properties of the porous medium. The current paper attempts to assess this impact on the computed bulk permeability of the medium.

We used a slip length formalism based on [19] and [32] to replace the actual rough surface by a smooth one, and/or the wall position subject to uncertainty due to image artefacts, supplemented with slip boundary conditions. The Stokes problem is then solved using a re-initiated GMRES method to ensure precise monitoring of the velocity at the pore interface, after which the permeability tensor is obtained using homogenization. Two approaches are proposed to calculate a range of values for the absolute permeability, namely full deviation and linear deviation. Bounds on the values are obtained by modeling Stokes flow with a no-slip boundary condition and a well-chosen slip condition.

Some non-linear effects appear as soon as $\beta$ nears the voxel size. At this point the permeability deviation and its estimation based on the linear deviation begin to differ. Nevertheless, within the range $\beta / h<1$, both the full deviation and the linear deviation have given comparable results. A formal derived problem is also provided to estimate the linear permeability deviation. Counter-intuitively, this problem does not involve a Navier boundary condition, but a prescribed slip (non-homogeneous Dirichlet condition).

The absolute permeability analysis on real porous rock applications have highlighted that a geometrical uncertainty may induce a significant deviation that can result in twice the reference permeability. It must be noted that the permeability deviation is sensitive to the structure of the pore space and its effect increases for geometries with a large proportion of small pores. Consequently, we must be careful with the permeability estimation in which the geometrical uncertainty should be rendered by a range of permeability from tomographic datasets.

The resulting permeability ranges for our different samples are summarized in table 1. Representative samples have been shown to exhibit similar dimensionless $L_{0} / A_{s} K_{0}$ ratios in the range 12-17. This ratio quantifies the relative permeability deviation versus the specific area. It has also been observed to be close to the theoretical value of 2 for a non-representative single cylindrical pore. This result was obtained for a few samples and will be investigated in future work. Another prospect could be to extend the approach developed in this paper to multi-phase flows at low Reynolds regime, in order to monitor the relative permeability to each phase. 


\section{Credits}

The conceptualisation was developed by PP and SP. The computational code and HPC were implemented by SP. The article was written collectively by SP, PM and PP. The positioning and results were discussed collectively. The geometry and microCT scans were provided by PM. The mathematical analysis was performed by SP. Funding was provided by PP.

\section{Acknowledgements}

This work was partially supported by E2S UPPA and by the Carnot Institute ISIFoR with grantMicroMineral (Contract P450902ISI). For this work, the team was granted access to Pyrene's HPC resources (UPPA Cluster).

\section{A Numerical method}

Many numerical methods exist to solve the Stokes equation in a domain with complex morphology. We distinguish mesh-based methods from grid-based methods. Mesh-based methods include finite elements (FEM, [41, 42]), finite volumes (FV, [43, 44, 45]), Lattice Boltzmann (LBM, [12]), ALE [46] or Boundary Element methods [47, 48, 49]. Grid-based methods involve discrete operators on structured grids such as finite differences, staggered [50] or not [40], spectral methods [51], or FV [52] and LBM [53] on structured meshes. For successive evaluations of differential operators (typically gradient and divergence), grid-based methods can either use a collocation formulation or staggered grids. While the latter allow a gain in accuracy, the use of collocated grids easily aligns the computational points to the experimental datasets. Optimized grid-based methods may be coupled to other methods dedicated to the flow features to be investigated: transport based on particle methods [54, 55], anisotropic diffusion for space-variable medium [56, 57], phase-field description of multi-phase flows $[58,59]$ and its upscaling $[8,60]$, complex fluid and rheology [61, 57], etc...

In the present work, we consider a grid-based approximation of the stationary Stokes problem, with the velocity and pressure field as unknowns, using the restarted Generalized Minimal Residual Method (GMRES) the solve the linear system inherited from the centered and one-sided finite difference schemes. In this formalism, detailed hereafter, we encode and satisfy the Navier or Dirichlet boundary condition at the fluid/solid interface, the Stokes equation in the fluid region and a trivial identity inside the solid region, since these points are not connected to the fluid plus interface set of grid points.

We introduce a uniform Cartesian grid, with a mesh step of $h$, and $N_{x}, N_{y}$ and $N_{z}$ points in each direction. We also use a renumbering index to convert from 3D numbering, with respect to the indices $i, j$ and $k$ representing the grid points, to 1D numbering $l=i+N_{x}\left(j+N_{y} k\right)$. As stated before, such raw datasets can be segmented to obtain the characteristic function $\chi_{S}$ on the Cartesian grid points.

Next, we construct a discrete version of the continuous solid/liquid interface. To do so, we identify the mesh points $X_{i j k}=\left(x_{i}, y_{j}, z_{k}\right)$ which lie in the solid matrix and have at least one neighbour in the pore domain. We call this set $\Sigma_{s}$ and choose to impose the pore interface boundary conditions on this interface layer.

Let us define the unknowns vector $x \in \mathbb{R}^{4 N}$, with $N=N_{x} N_{y} N_{z}$ the total number of mesh points, such that $x=\left(u_{x}, u_{y}, u_{z}, p\right)^{T}$. Then, depending on the mesh point identification, we define the numerical product function $A x$, from $\mathbb{R}^{4 N}$ to $\mathbb{R}^{4 N}$, representing the discretization of equation (3) extended by 0 for both the velocity and the pressure inside the solid domain, as follows:

$$
A x\left(X_{i j k}\right)= \begin{cases}-\Delta_{h} u\left(X_{i j k}\right)+\nabla_{h} p\left(X_{i j k}\right), & \text { if } \chi_{s}\left(X_{i j k}\right)=0, \\ \operatorname{div}_{h} u\left(X_{i j k}\right), & \left(\Longleftrightarrow X_{i j k} \in \Omega_{F}\right) \\ u\left(X_{i j k}\right) \cdot \tau_{k}\left(X_{i j k}\right)-\beta D_{h}(u) n\left(X_{i j k}\right) \cdot \tau_{k}\left(X_{i j k}\right), & \text { if } X_{i j k} \in \Sigma_{s} \\ u\left(X_{i j k}\right) \cdot n\left(X_{i j k}\right), & \\ \operatorname{div}_{h} u\left(X_{i j k}\right), & \\ u\left(X_{i j k}\right), & \text { otherwise. } \\ p\left(X_{i j k}\right), & \end{cases}
$$

where the index $h$ denotes the usual discrete operators defined above.

The evaluation of the differential operators, including Laplacian, divergence and gradient, is performed using straightforward finite difference schemes of order two. The formalism used in this study is based on the fact that the solid and fluid domains are disconnected and can be considered as two independent parts in the linear system. We are therefore interested in decoupling the problem between the solid on the one hand, and the fluid+interface parts on the other hand, such that the computational size of the problem could be reduced to just the latter. Consequently, we use centered schemes in the whole fluid part, even for the interface neighbors, and non-centered schemes in the normal direction, towards the fluid, on the first solid layer $\Sigma_{s}$. 


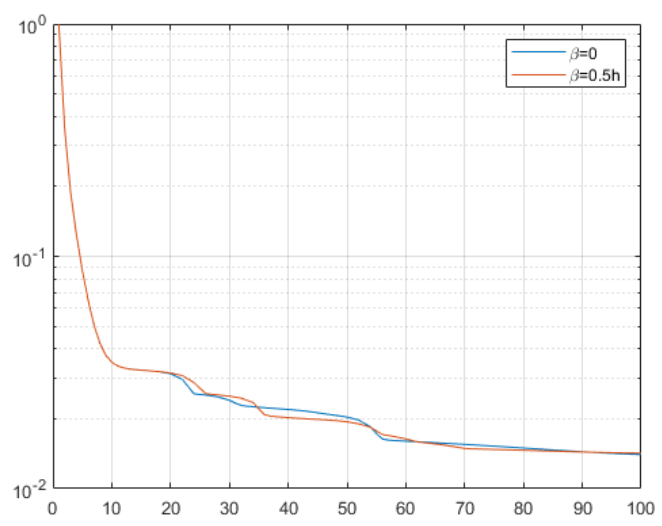

(a) Relative residual norm, Bentheimer sandstone

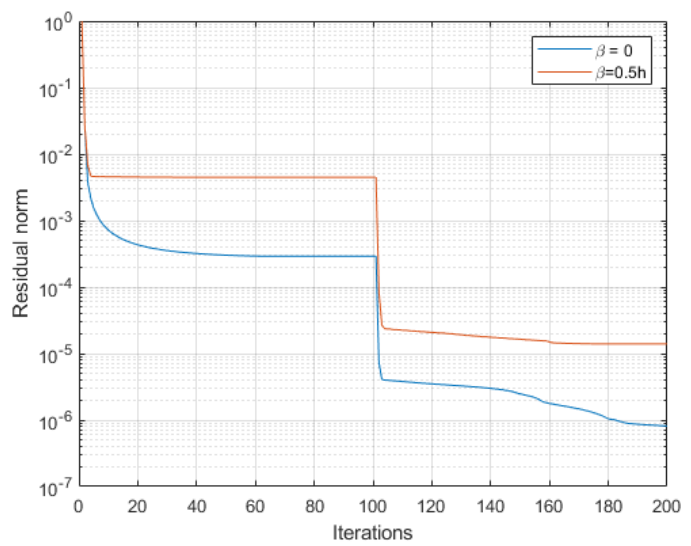

(c) Relative residual norm, Castlegate sandstone

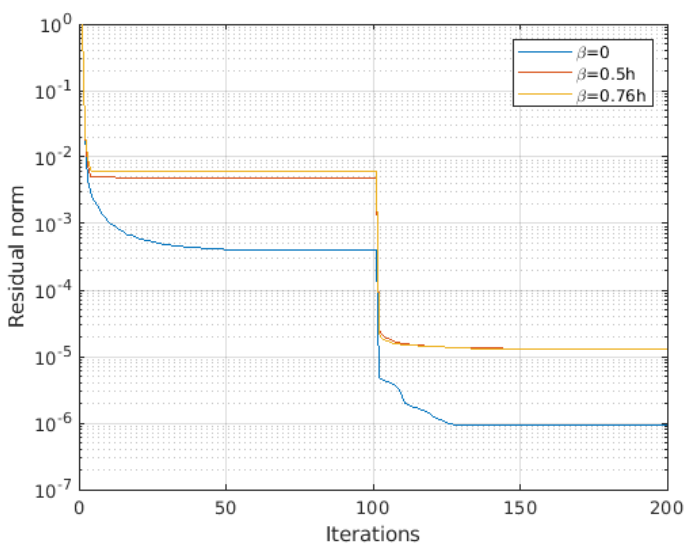

(e) Relative residual norm, sandpack

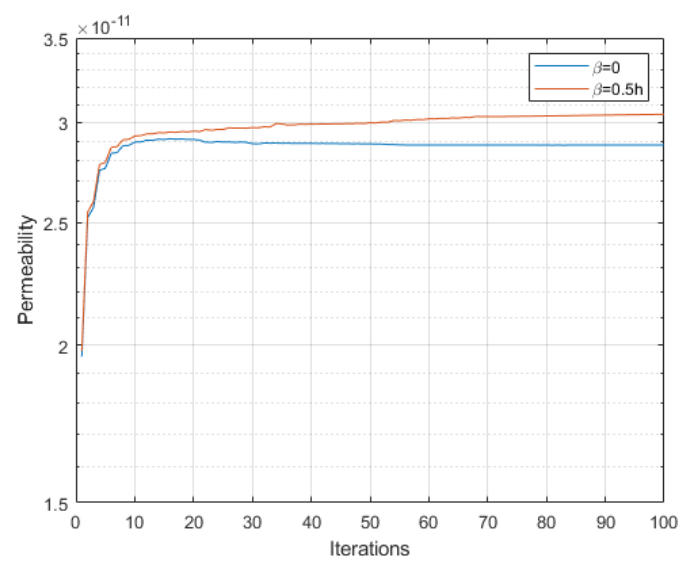

(b) Permeability evolution, Bentheimer sandstone

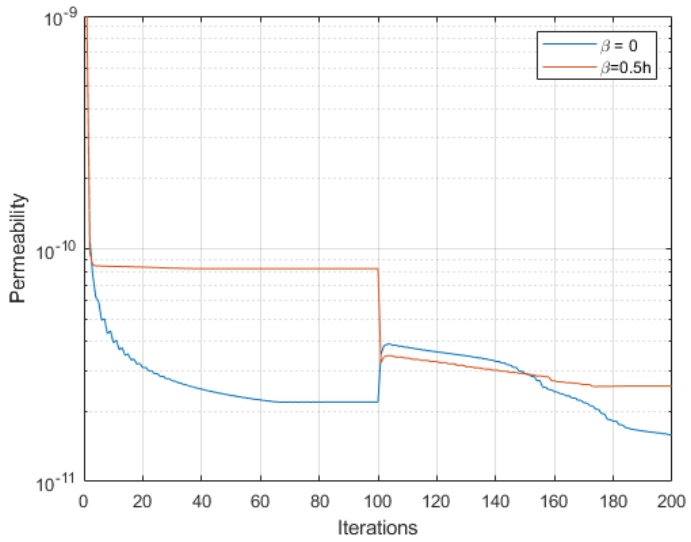

(d) Permeability evolution, Castlegate sandstone

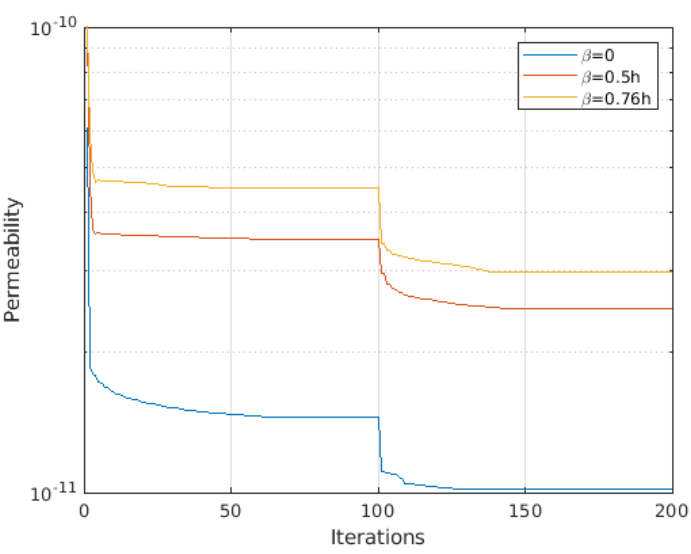

(f) Permeability evolution, sandpack

Fig. 15 Relative residual norm (left pictures) quantifying the convergence of the numerical method, and the evolution of the permeability estimation with respect to the iterations of the numerical method (right pictures), for the different samples: the Bentheimer geometry for $\beta / h=0$ and 0.5 to the top, the Castlegate sandstone sample using preconditioning with $2^{8}$ grid size until the 100 th iteration for $\beta / h=0$ and 0.5 at the middle, and the Sand pack sample also using preconditioning, for $\beta / h=0,0.5$ and 0.76 .

Concerning the right-hand-side of the problem, denoted by $\left(f_{x}, f_{y}, f_{z}\right) \in \mathbb{R}^{3 N}$ the components of the driving force $f$, we have the following expressions for $b$ :

$$
b\left(X_{i j k}\right)= \begin{cases}\left(f_{x}, f_{y}, f_{z}, 0\right), & \text { if } \chi_{s}\left(X_{i j k}\right)=0 \\ (0,0,0,0), & \text { otherwise }\end{cases}
$$

noting that the last components of the vector $b$ ensure the incompressibility condition, on the fluid and first solid layer parts. On the solid part, it also imposes a value of zero for both the velocity field and the pressure. Moreover, this method presents the advantage that it algebraically ensures the divergence-free condition. 
We use the GMRES method to solve the linear system $A x=b$ introduced above. It was originally developed in 1986 by Saad and Schultz [62] and we go over the general principles hereafter. GMRES is an iterative method that aims at solving a non-symmetric system of linear equations, with $A \in \mathbb{R}^{N \times N}$ a non-symmetric square matrix and $b \in \mathbb{R}^{N}$.

The method approximates the solution by a vector in a Krylov subspace with a minimal residual. We define the Krylov subspace of order $m$ related to this problem with respect to the initial residual $r_{0}$ by

$$
\mathcal{K}_{m}\left(A, r_{0}\right)=\operatorname{Span}\left\{r_{0}, A r_{0}, \ldots, A^{m-1} r_{0}\right\}
$$

where $r_{0}=b-A x_{0}$ and $x_{0} \in \mathbb{R}^{N}$ is the initial iterate.

The GMRES algorithm provides an approximate solution that minimizes, at every time step, the Euclidean norm denoted by $\|$.$\| of the residual vector. The next iterate x_{k}$ is sought in the form $x_{k}=x_{0}+z_{k}$ where $z_{k} \in \mathcal{K}_{k}\left(A, r_{0}\right)$ is the solution of the least squares problem:

$$
\min _{z \in \mathcal{K}_{k}\left(A, r_{0}\right)}\left\|b-A\left(x_{0}+z\right)\right\|=\min _{z \in \mathcal{K}_{k}\left(A, r_{0}\right)}\left\|r_{0}-A z\right\| .
$$

First, the modified Gram-Schmidt Arnoldi algorithm is applied to build an orthonormal basis $\left\{v_{1}, \ldots, v_{k}\right\}$ for the Krylov subspace $\mathcal{K}_{k}\left(A, r_{0}\right)$. It provides the following relationship:

$$
A V_{k}=V_{k+1} \bar{H}_{k}
$$

where $\bar{H}_{k} \in \mathbb{R}^{(k+1) \times k}$ is an upper Hessenberg matrix whose only non-zero entries are the $h_{i j}$ elements generated by the Arnoldi process, and $V_{k+1} \in \mathbb{R}^{N \times(k+1)}$ is an orthogonal matrix whose columns are the orthonormal basis $\left\{v_{1}, \ldots, v_{k+1}\right\}$. Using this relation, it has been shown in [62] that by setting $z=V_{k} y$ the solution of the problem (21) is $x_{k}=x_{0}+V_{k} y_{k}$, with $y_{k}$ the solution of

$$
\min _{y \in \mathbb{R}^{k}}\left\|\beta e_{1}-\bar{H}_{k} y\right\|,
$$

where $\beta=\left\|r_{o}\right\|$ and $e_{1}$ is the first canonical vector of $\mathbb{R}^{k+1}$. The above problem is generally solved (Givens) using a $Q R$ decomposition of the Hessenberg matrix.

Among the main convergence results of the GMRES method, we reiterate the following proposition from [62]:

Property 1 Let A be diagonalizable such that $A=X D X^{-1}$ and we denote $P_{m}$ the space of all polynomials of degree $\leq m$ and $\sigma$ the spectrum of A. Then, the residual norm provided at the $m^{t h}$ step of GMRES satisfies

$$
\left\|r_{m+1}\right\| \leq\|X\|\left\|X^{-1}\right\| \varepsilon^{(m)}\left\|r_{0}\right\|
$$

with $\varepsilon^{(m)}=\min _{p \in P_{m}, p(0)=1} \max _{\lambda_{i} \in \sigma}\left|p\left(\lambda_{i}\right)\right|$.

The convergence of the restarted GMRES(m) algorithm is also proven when A is a positive real with an error bound derived from the above proposition. However, this result is not consistent when A is not positive real, in which case the following theorem has been proven:

Property 2 Assuming that there are $\nu$ eigenvalues $\lambda_{1}, \lambda_{2}, \ldots, \lambda_{\nu}$ of A with non-positive real parts. Let the other eigenvalues be enclosed in a circle centered on $C$ with $C>0$ and with a radius $R$ where $C>R$. Then,

$$
\varepsilon^{(m)} \leq\left(\frac{R}{C}\right)^{m-\nu} \max _{j=\nu+1, N} \prod_{i=1}^{\nu} \frac{\left|\lambda_{i}-\lambda_{j}\right|}{\left|\lambda_{i}\right|} \leq\left(\frac{D}{d}\right)^{\nu}\left(\frac{R}{C}\right)^{m-\nu}
$$

with $D=\max _{i=1, \nu ; j=\nu+1, N}\left|\lambda_{i}-\lambda_{j}\right|$ and $d=\min _{i=1, \nu}\left|\lambda_{i}\right|$.

In any case, the convergence is closely linked to the condition number of $X$ and the eigenvalue distribution: in particular, extremely small eigenvalues slow down the convergence.

In this study, the stop criteria for the convergence is given with respect to the relative residual norm at the iterate $x_{k}$ such that

$$
\left\|r_{k}\right\|=\frac{\left\|b-A x_{k}\right\|}{\left\|b-A x_{0}\right\|}<\varepsilon
$$

for a given tolerance $\varepsilon$, but it also includes a maximum number of iterations for the restarted algorithm.

Regarding memory requirements, the cost of the method rises as the Krylov subspace grows so in practice restarted methods are used to reduce the storage and orthogonalization costs. This means that the algorithm is restarted every $m$ steps, with $m$ a fixed integer that sets the Krylov subspace size, and the initial guess chosen for the restart is the final previous one, obtained by minimizing the residual norm. Furthermore, we do not use an assembly procedure for the matrix $A$ but rather focus on the implementation of a matrix-vector product function. From a numerical point of view, we use a Fortran subroutine for the GMRES restarted method and a $\mathrm{C}$ implementation of the main program including the definition of the matrix-vector product function computing $A x$, with $A$ the matrix representing the Stokes problem and $x$ the main unknowns vector, which contains the velocity and pressure fields.

The relative residual errors and convergence of the permeability values are displayed in Figure 15 for the three different samples analyzed in section 4 . 


\section{B Preconditioning and interpolation method}

Regarding a sample geometry which contains various small pores, we consider the preconditioning on a grid twice as coarse, for example a grid size of $2^{9}$ cells per direction inherited from a $2^{8}$ one.

We perform an interpolation of these fields to obtain their approximation on the finer final grid using an interpolation kernel and then the interpolated pressure and velocity fields are taken as initial data for the computation on the whole grid. In the remainder, we use tilde notations when dealing with the refined grid variables and fields, so we denote the interpolated velocity and pressure by $\tilde{u}$ and $\tilde{p}$, respectively. We also respectively introduce $\tilde{x}_{q}$ and $x_{p}$ in $\mathbb{R}^{3}$ as the space variables on the refined and coarse grids. Afterwards, the interpolated velocity is computed using the convolution:

$$
\tilde{u}\left(\tilde{x}_{q}\right)=\left(u * M_{4}^{\prime \varepsilon}\right)\left(\tilde{x}_{q}\right)=\int_{\mathbb{R}^{3}} u(x) M_{4}^{\prime \varepsilon}\left(\tilde{x}_{q}-x\right) d x=\sum_{p \in S} u_{p} M_{4}^{\prime \varepsilon}\left(\tilde{x}_{q}-x_{p}\right) v_{p}
$$

where $S=\left\{p\right.$ s.t $\left.\tilde{x}_{q}-x_{p} \in \operatorname{supp}\left(M_{4}^{\prime \varepsilon}\right)\right\}$ and $v_{p}$ the volume element of the coarse grid. Moreover, $M_{4}^{\prime \varepsilon}$ is defined with respect to the classical $1 \mathrm{D}$ interpolation kernel $M_{4}^{\prime}$

$$
M_{4}^{\prime}(x)=\left\{\begin{array}{cc}
\left(3 x^{3}-5 x^{2}+2\right) / 2 & \text { if } 0 \leq x \leq 1 \\
(2-x)^{2}(1-x) / 2 & \text { if } 1 \leq x \leq 2 \\
0 & \text { if } x \geq 2
\end{array}\right.
$$

and is written as the following tensor product, with $\varepsilon$ the coarse grid mesh step:

$$
M_{4}^{\prime \varepsilon}(x)=\frac{1}{\varepsilon^{3}} M_{4}^{\prime \otimes 3}\left(\frac{x}{\varepsilon}\right) .
$$

\section{Asymptotic expansion of the Stokes equation with slip}

We denote by $\varphi: \Omega_{F} \rightarrow \mathbb{R}^{+}$the euclidean distance to the boundary $\varphi(x)=\operatorname{dist}(x, \Sigma)$. Assuming sufficient regularity on $\Omega_{F}$, typically a smooth bounded open set, $\varphi$ is smooth in the neighborhood $\omega \subset \Omega_{F}$ of $\Sigma$. In addition, it can be shown using differential geometry tools that $\forall x \in \omega,\|\nabla \varphi\|=1$ and $\forall x \in \Sigma, \nabla \varphi(x)=n(x)$, where we recall that $n$ is the inward unit normal at the interface, oriented towards the fluid region. We can also extend $n$ by setting $n(x)=\nabla \varphi(x)$. As a formal step, we look for the velocity and the pressure in the usual asymptotic expansion form:

$$
\begin{array}{ll}
u_{\beta}(x) \simeq \sum_{j \geq 0} \beta^{j} U^{j}\left(x, \frac{\varphi(x)}{\beta}\right), & \text { with } U^{j}(x, z)=\bar{U}^{j}(x)+\widetilde{U}^{j}(x, z) \\
p_{\beta}(x) \simeq \sum_{j \geq 0} \beta^{j} P^{j}\left(x, \frac{\varphi(x)}{\beta}\right), & \text { with } P^{j}(x, z)=\bar{P}^{j}(x)+\widetilde{P}^{j}(x, z)
\end{array}
$$

where the boundary layer terms $\widetilde{U}^{j}$ and $\widetilde{P}^{j}$ and their derivatives must tend to 0 when $z \rightarrow+\infty$ and the interior terms $\bar{U}^{j}$ and $\bar{P}^{j}$ depend only on $x$, that is at the limit of $U^{j}$ and $P^{j}$ when $z \rightarrow+\infty$. We plug the asymptotic expansions (29a) and (29b) in the equations above and formally assume that $x$ and $z$ are independent to identify the powers of $\beta$ to thereafter characterize the profiles $U^{j}$ and $P^{j}$, for $j=0$ and 1 .

In order to differentiate the main operators involved, we introduce a generic function $\psi$, whose values belong to $\mathbb{R}^{3}$ and $\mathbb{R}$ for the velocity and pressure profiles respectively, defined by $\psi(x)=\Psi\left(x, \frac{\varphi(x)}{\beta}\right)$. Simple calculations of the first derivatives provide for $x \in \mathbb{R}^{3}$ :

$$
\frac{\partial \psi}{\partial x_{j}}(x)=\frac{\partial \Psi}{\partial x_{j}}\left(x, \frac{\varphi(x)}{\beta}\right)+\frac{1}{\beta} \frac{\partial \Psi}{\partial z}\left(x, \frac{\varphi(x)}{\beta}\right) \frac{\partial \varphi}{\partial x_{j}}(x)
$$

which can be written using standard notations as

$$
\nabla \psi=\beta^{-1} \nabla \varphi \partial_{z} \Psi+\nabla \Psi
$$

In the same way, we can obtain the other operators as follows:

$$
\begin{aligned}
\Delta \psi & =\beta^{-2}\|\nabla \varphi\|^{2} \partial_{z z} \Psi+\beta^{-1}\left(2 \nabla \varphi \cdot \nabla \partial_{z} \Psi+\Delta \varphi \partial_{z} \Psi\right)+\Delta \Psi \\
\operatorname{div} \psi & =\beta^{-1} \nabla \varphi \cdot \partial_{z} \Psi+\operatorname{div} \Psi \\
D(\psi) \cdot n & =\beta^{-1} \frac{1}{2}\left(\partial_{z} \Psi+\left(\partial_{z} \Psi \cdot n\right) n\right)+D(\Psi) \cdot n, \quad \text { on } \Sigma .
\end{aligned}
$$

The terms with the same power in $\beta$ are identified for each equation of (9). We first consider (9a). 
At order $\beta^{-2}$, it holds that $-\partial_{z z} U^{0}(x, z)=-\partial_{z z} \widetilde{U}^{0}(x, z)=0$ with the assumption that $\widetilde{U}^{0}(x, z) \rightarrow 0$ when $z \rightarrow+\infty$. Thus we get $\widetilde{U}^{0}=0$ in $\Omega_{F} \times \mathbb{R}^{+}$and

$$
U^{0}(x, z)=\bar{U}^{0}(x) .
$$

At order $\beta^{-1}$ and considering directly that $\widetilde{U}^{0}=0$, we infer that

$$
-\partial_{z z} U^{1}(x, z)+\nabla \varphi \partial_{z} P^{0}(x, z)=0 .
$$

At order $\beta^{0}$, we find that $-\Delta U^{0}-2 \nabla \varphi \cdot \nabla \partial_{z} U^{1}-\Delta \varphi \partial_{z} U^{1}-\partial_{z z} U^{2}+\nabla P^{0}+\nabla \varphi \partial_{z} P^{1}=f$, hence taking the limit $z \rightarrow+\infty$ and based on the assumption that tilda terms and all their derivatives tend to 0 when $z$ tends to $+\infty$, we find again the Stokes equation

$$
-\Delta U^{0}+\nabla P^{0}=f, \quad \text { in } \Omega_{F}
$$

and by taking the difference with the previous, we also have

$$
-\partial_{z z} U^{2}-2 \nabla \varphi \cdot \nabla \partial_{z} U^{1}-\Delta \varphi \partial_{z} U^{1}+\nabla \varphi \partial_{z} P^{1}=0, \quad \text { in } \Omega_{F} \times \mathbb{R}^{+} .
$$

The latter can be generalized at any order $k \geq 1$ given the following set of equations

$$
\begin{gathered}
-\Delta U^{k}+\nabla P^{k}=0, \\
-\partial_{z z} U^{k+2}-2 \nabla \varphi \cdot \nabla \partial_{z} U^{k+1}-\Delta \varphi \partial_{z} U^{k+1}+\nabla \varphi \partial_{z} P^{k+1}=0 .
\end{gathered}
$$

For equation (9b) at order $\beta^{0}$, we get $\operatorname{div} U^{0}+\nabla \varphi \cdot \partial_{z} U^{1}=0$, noting that the term in $\beta^{-1}$ involving only $\tilde{U}^{0}$ vanishes with (35). Taking the limit $z$ tends to $+\infty$, we infer the two relations: $\operatorname{div} U^{0}=0$ in $\Omega_{F}$ first and then, $\nabla \varphi \cdot \partial_{z} U^{1}=0$. For the latter, we take $\nabla \varphi=n$ and assume that $\widetilde{U}^{1}(x, z) \rightarrow 0$ when $z \rightarrow+\infty$ to obtain $\widetilde{U}^{1} \cdot n=0$ in $\Omega_{F} \times \mathbb{R}^{+}$. We can easily generalize and thus the divergence-free condition provides the following set of equations for $k \geq 0$ :

$$
\begin{gathered}
\operatorname{div} U^{k}=0, \quad \text { in } \Omega_{F} \\
\widetilde{U}^{k+1} \cdot n=0 .
\end{gathered}
$$

For equation (9d), we directly obtain $U^{k} \cdot n=0$ on $\Sigma$ for $k \geq 0$ and (9c) at order $\beta^{0}$ provides $U^{0}+\frac{1}{2}\left(\partial_{z} U^{0}+\left(\partial_{z} U^{0} \cdot n\right) n\right)=$ 0 which according to (35) reduces to

$$
U^{0}(x, 0)=\bar{U}^{0}(x)=0, \quad \text { for } x \in \Sigma .
$$

Finally, at any order $k \geq 1$ we can obtain:

$$
U^{k}(x, 0)+\frac{1}{2}\left(\partial_{z} U^{k}(x, 0)+\left(\partial_{z} U^{k}(x, 0) \cdot n\right) n\right)=D\left(U^{k-1}\right) \cdot n, \quad \text { for } x \in \Sigma .
$$

At this step, we are able to characterize the profiles $U^{j}$ and $P^{j}$. We have already obtained from equations (37), (41), (43) that $\left(U^{0}, P^{0}\right)$ satisfy a no-slip Stokes problem with $U^{0}(x, z)=\bar{U}^{0}(x)$ and $P^{0}(x, z)=\bar{P}^{0}(x)$ for any $z$. Consequently the functions $\left(U^{0}, P^{0}\right)$ are reduced to one space variable and satisfy the no-slip Stokes problem

$$
\begin{cases}-\Delta U^{0}+\nabla P^{0}=f, & \text { in } \Omega_{F} \\ \operatorname{div} U^{0}=0, & \text { in } \Omega_{F} \\ U^{0}=0, & \text { on } \Sigma\end{cases}
$$

Then, the tangential component of (36) reduces to $-\partial_{z z} T\left(U^{1}\right)=0$ (as a reminder $T$ is the projection operator on the tangential components as defined in section 2.1). Hence, in the same way as before, we obtain $T\left(\widetilde{U}^{1}\right)=0$. Combined with (42) for $k=0$ we have $\widetilde{U}^{1}=0$ in $\Omega_{F} \times \mathbb{R}^{+}$(since the tangential and normal parts are both equal to 0 ). On the normal part of (36), there remains $\nabla \varphi \partial_{z} P^{0}=0$ which gives, since $\widetilde{P}^{0}(x, z)=0$ for any $z, \widetilde{P}^{0}=0$ and thus $P^{0}(x, z)=\bar{P}^{0}(x)$, as announced in the definition of the problem (10). Furthermore, equation (38) can now reduce to $-\partial_{z z} U^{2}+\nabla \varphi \partial_{z} P^{1}=0$ which resembles equation (36), so we can check in the same way that $\widetilde{P}^{1}=0$.

Taking $k=1$ in equations (39), (41) and (44), we obtain profiles $\left(U^{1}, P^{1}\right)$ that satisfy the following Stokes problem with slip: there are functions $\bar{U}^{1}$ and $\bar{P}^{1}$ such as $U^{1}(x, z)=\bar{U}^{1}(x)$ and $P^{1}(x, z)=\bar{P}^{1}(x)$ such that the functions $\left(U^{1}, P^{1}\right)$ are reduced to one variable of space and satisfy

$$
\begin{cases}-\Delta U^{1}+\nabla P^{1}=0, & \text { in } \Omega_{F} \\ \operatorname{div} U^{1}=0, & \text { in } \Omega_{F} \\ T U^{1}=T D\left(U^{0}\right) \cdot n, & \text { on } \Sigma \\ U^{1} \cdot n=0, & \text { on } \Sigma .\end{cases}
$$

Assuming sufficient regularity on $\Omega_{F}$, supposed to be simply connected, and regularity of the source function $f$, then problems (45) and (11) (from the main text) admit unique weak solutions, up to additive constants for $P^{0}$ and $P^{1}$, and satisfy regularity results, not developed here. We refer to the De Rahm theorem and to the elliptic regularity results for the existence, uniqueness and regularity demonstrations of such variational problems. Finally, one would also have to characterize the remainder and provide its second order estimation, so that the first order asymptotic expansion can be strictly justified. 


\section{References}

1. W. Benton, Encyclopaedia Britannica. University of Chicago Press, 1963.

2. D. Wildenschild and A. Sheppard, "X-ray imaging and analysis techniques for quantifying pore-scale structure and processes in subsurface porous medium systems," Advances in Water Resources, vol. 51, pp. 217-246, 2013.

3. N. Saxena, R. Hofmann, F. O. Alpak, J. Dietderich, S. Hunter, and R. J. Day-Stirrat, "Effect of image segmentation and voxel size on micro-CT computed effective transport \& elastic properties," Marine and Petroleum Geology, vol. 86, pp. 972-990, Sept. 2017.

4. Y. Mehmani and H. Tchelepi, "Pore-Network Modeling vs. Direct Numerical Simulation: a Comparative Study," in AGU Fall Meeting Abstracts, vol. 2016, pp. H51D-1519, Dec. 2016.

5. S. Schlüter, A. Sheppard, K. Brown, and D. Wildenschild, "Image processing of multiphase images obtained via x-ray microtomography: A review,” Water Resources Research, vol. 50, no. 4, pp. 3615-3639, 2014.

6. J. Banhart, Advanced Tomographic Methods in Materials Research and Engineering. Monographs on the Physics and Chemistry of Materials, Oxford: Oxford Univ. Press, 2008.

7. C. Soulaine, F. Gjetvaj, C. Garing, S. Roman, A. Russian, P. Gouze, and H. A. Tchelepi, "The Impact of SubResolution Porosity of X-ray Microtomography Images on the Permeability," Transport in Porous Media, vol. 113, no. 1, pp. 227-243, 2016.

8. D. Lasseux, F. J. V. Parada, and M. L. Porter, "An improved macroscale model for gas slip flow in porous media," Journal of Fluid Mechanics, vol. 805, pp. 118-146, Sept. 2016.

9. D. Lasseux, F. J. Valdés-Parada, and A. Bottaro, "Upscaled model for unsteady slip flow in porous media," Journal of Fluid Mechanics, vol. 923, Aug. 2021.

10. W. P. Breugem, B. J. Boersma, and R. E. Uittenbogaard, "The influence of wall permeability on turbulent channel flow," Journal of Fluid Mechanics, vol. 562, p. 35-72, 2006.

11. K. Suga, Y. Matsumura, Y. Ashitaka, S. Tominaga, and M. Kaneda, "Effects of wall permeability on turbulence," International Journal of Heat and Fluid Flow, vol. 31, no. 6, pp. 974 - 984, 2010. 7th World Conference on Experimental Heat Transfer, Fluid Mechanics and Thermodynamics (ExHFT-7), Krakow and The Conference on Modelling Fluid Flow (CMFF' 09), Budapest.

12. S. Molins, C. Soulaine, N. I. Prasianakis, A. Abbasi, P. Poncet, A. J. C. Ladd, V. Starchenko, S. Roman, D. Trebotich, H. A. Tchelepi, and C. I. Steefel., "Simulation of mineral dissolution at the pore scale with evolving fluid-solid interfaces: Review of approaches and benchmark problem set," Computational Geosciences, 2020, https://doi.org/10.1007/s10596-019-09903-x.

13. E. Lauga and M. P. Brenner, "Dynamic mechanisms for apparent slip on hydrophobic surfaces," Physical Review $E$, vol. 70, no. 2, p. 026311, 2004-08-31.

14. E. Secchi, S. Marbach, A. Niguès, D. Stein, A. Siria, and L. Bocquet, "Massive radius-dependent flow slippage in carbon nanotubes," Nature, vol. 537, pp. 210-213, Sept. 2016.

15. S. Berg, A. Cense, J. Hofman, and R. Smits, "Two-phase flow in porous media with slip boundary condition," Transport in porous media, vol. 74, no. 3, pp. 275-292, 2008.

16. P.-G. de Gennes, “On fluid/wall slippage,” Langmuir, vol. 18, no. 9, pp. 3413-3414, 2002.

17. C. L. M. H. Navier, "Mémoire sur les lois du mouvement des fluides," Mémoires de l'Académie Royale des Sciences de l'Institut de France, vol. 6, no. 1823, pp. 389-440, 1823.

18. Y. Achdou, O. Pironneau, and F. Valentin, "Effective boundary conditions for laminar flows over periodic rough boundaries," Journal of Computational Physics, vol. 147, pp. 187-218, 1998.

19. E. Lauga and H. A. Stone, "Effective slip in pressure-driven stokes flow," Journal of Fluid Mechanics, vol. 489, p. $55,2003$.

20. S. Pasquier, M. Quintard, and Y. Davit, "Modeling flow in porous media with rough surfaces: effective slip boundary conditions and application to structured packings," Chemical Engineering Science, vol. 165, pp. 131-146, 2017.

21. J. Davies, D. Maynes, B. W. Webb, and B. Woolford, "Laminar flow in a microchannel with superhydrophobic walls exhibiting transverse ribs," Physics of fluids, vol. 18, no. 8, p. 087110, 2006.

22. A. V. Belyaev and O. I. Vinogradova, "Effective slip in pressure-driven flow past super-hydrophobic stripes," Journal of Fluid Mechanics, vol. 652, pp. 489-499, 2010.

23. P. Joseph, C. Cottin-Bizonne, J.-M. Benoît, C. Ybert, C. Journet, P. Tabeling, and L. Bocquet, "Slippage of water past superhydrophobic carbon nanotube forests in microchannels," Phys. Rev. Lett., vol. 97, p. 156104, Oct 2006.

24. A. M. Davis and E. Lauga, "Hydrodynamic friction of fakir-like super-hydrophobic surfaces," Journal of Fluid Mechanics, vol. 661, pp. 402-411, 2010. 
25. C. Ybert, C. Barentin, C. Cottin-Bizonne, P. Joseph, and L. Bocquet, "Achieving large slip with superhydrophobic surfaces: Scaling laws for generic geometries," Physics of fluids, vol. 19, no. 12, p. 123601, 2007.

26. C. Lee, C.-H. Choi, and C.-J. Kim, "Superhydrophobic drag reduction in laminar flows: a critical review," Experiments in Fluids, vol. 57, no. 12, p. 176, 2016.

27. W. Degruyter, O. Bachmann, and A. Burgisser, "Controls on magma permeability in the volcanic conduit during the climactic phase of the kos plateau tuff eruption (aegean arc)," Bulletin of Volcanology, vol. 72, no. 1, pp. 63-74, 2010. cited By 59.

28. C. Noiriel, C. Steefel, L. Yang, and D. Bernard, "Effects of pore-scale precipitation on permeability and flow," Advances in Water Resources, vol. 95, pp. 125-137, 2016.

29. M. Christensen and Y. Tanino, "Enhanced permeability due to apparent oil/brine slippage in limestone and its dependence on wettability," Geophysical Research Letters, vol. 44, no. 12, pp. 6116-6123, 2017.

30. K. Daly and T. Roose, "Multiscale modelling of hydraulic conductivity in vuggy porous media," Proceedings of the Royal Society A: Mathematical, Physical and Engineering Sciences, vol. 470, no. 2162, 2014. cited By 12.

31. P. Di Palma, N. Guyennon, F. Heße, and E. Romano, "Porous media flux sensitivity to pore-scale geostatistics: A bottom-up approach," Advances in Water Resources, vol. 102, pp. 99-110, 2017. cited By 10.

32. M. Bonnivard, A.-L. Dalibard, and D. Gérard-Varet, "Computation of the effective slip of rough hydrophobic surfaces via homogenization," Mathematical Models and Methods in Applied Sciences, vol. 24, p. 2259-2285, 2014.

33. M. Quintard and S. Whitaker, "Two phase flow in heterogeneous porous media: the method of large-scale averaging," Transport in Porous Media, vol. 3, pp. 357-413, 1987.

34. A. Sheppard and M. Prodanovic, "Network generation comparison forum." https://www.digitalrocksportal.org/projects/16, 2015.

35. T. Yao, B. A. Baudet, and S. D. N. Lourenço, "Quantification of the surface roughness of quartz sand using optical interferometry," Meccanica, vol. 54, pp. 741-748, July 2018.

36. D. Krinsley and P. Trusty, "Environmental interpretation of quartz grain surface textures," in Provenance of Arenites, pp. 213-229, Springer Netherlands, 1985.

37. P. Andriamananjaona, Wettability in porous media - From macroscopic measurements to pore-scale characterization. PhD thesis, Université de Pau et des Pays de l'Adour, 2020.

38. Z. Zhang, S. Kruschwitz, A. Weller, and M. Halisch, "Enhanced pore space analysis by use of $\mu$-CT, MIP, NMR, and SIP," Solid Earth, vol. 9, pp. 1225-1238, 112018.

39. A. E. Peksa, K.-H. A. Wolf, and P. L. Zitha, "Bentheimer sandstone revisited for experimental purposes," Marine and Petroleum Geology, vol. 67, pp. 701-719, 2015.

40. L. Hume and P. Poncet, "A velocity-vorticity method for highly viscous $3 \mathrm{~d}$ flows with application to digital rock physics," Journal of Computational Physics, vol. 425, p. 109910, 2021.

41. L. Akanji and S. Matthai, "Finite element-based characterization of pore-scale geometry and its impact on fluid flow," Transp. Porous Med., vol. 81, p. 241-259, 2010.

42. M. Balhoff, S. Thomas, and M. Wheeler, "Mortar coupling and upscaling of pore-scale models," Computational Geosciences, vol. 12, pp. 15-27, 032008.

43. J. P. Pereira-Nunes, M. J. Blunt, and B. Bijeljic, "Pore-scale simulation of carbonate dissolution in micro-CT images," J. Geophys. Res. Solid Earth, vol. 121, pp. 558-576, 2016.

44. S. Molins, D. Trebotich, C. Steefel, and C. Shen, "An investigation of the effect of pore scale flow on average geochemical reaction rates using direct numerical simulation," Water Resour. Res., vol. 48, 2012.

45. C. Cancès, T. Gallouët, M. Laborde, and L. Monsaingeon, "Simulation of multiphase porous media flows with minimising movement and finite volume schemes," European Journal of Applied Mathematics, vol. 30, no. 6, p. 1123-1152, 2019.

46. V. Starchenko, C. J. Marra, and A. J. C. Ladd, "Three-dimensional simulations of fracture dissolution," Journal of Geophysical Research: Solid Earth, vol. 121, no. 9, pp. 6421-6444, 2016. _eprint: https://agupubs.onlinelibrary.wiley.com/doi/pdf/10.1002/2016JB013321.

47. S. Liska and T. Colonius, "A fast lattice green's function method for solving viscous incompressible flows on unbounded domains," Journal of Computational Physics, vol. 316, pp. 360 - 384, 2016.

48. P. Poncet, "Analysis of direct three-dimensional parabolic panel methods," SIAM Journal on Numerical Analysis, vol. 45, no. 6, pp. 2259-2297, 2007-01. Publisher: Society for Industrial and Applied Mathematics.

49. P. Barreau, D. Lasseux, H. Berlin, and A. Zaitowf, "Effect of adsorbed polymers on relative permeability and capillary pressure: a pore scale numerical study," WIT Transactions on Modelling and Simulation, vol. 10, 1970.

50. Y. Morinishi, T. Lund, O. Vasilyev, and P. Moin, "Fully conservative higher order finite difference schemes for incompressible flow," undefined, 1998. 
51. A. T. Patera, "A spectral element method for fluid dynamics: Laminar flow in a channel expansion," Journal of Computational Physics, vol. 54, no. 3, pp. 468-488, 1984.

52. C. Soulaine, S. Roman, A. Kovscek, and H. Tchelepi, "Mineral dissolution and wormholing from a pore-scale perspective," Journal of Fluid Mechanics, vol. 827, pp. 457 - 483, Sept. 2017.

53. S. Khirevich and T. Patzek, "Behavior of numerical error in pore-scale lattice boltzmann simulations with simple bounce-back rule: Analysis and highly accurate extrapolation,” Physics of Fluids, vol. 30, p. 093604, 2018.

54. U. Bandara, A. Tartakovsky, M. Oostrom, B. Palmer, J. Grate, and C. Zhang, "Smoothed particle hydrodynamics pore-scale simulations of unstable immiscible flow in porous media," Advances in Water Resources, vol. 62, no. Part C, pp. 356-369, 2013.

55. R. Chatelin and P. Poncet, "A hybrid grid-particle method for moving bodies in 3D stokes flow with variable viscosity," SIAM Journal on Scientific Computing, vol. 35, pp. B925-B949, Aug. 2013.

56. J.-M. Etancelin, P. Moonen, and P. Poncet, "Improvement of remeshed lagrangian methods for the simulation of dissolution processes at pore-scale," Advances in Water Resources, vol. 146, p. 103780, 2020.

57. D. Sanchez, L. Hume, R. Chatelin, and P. Poncet, "Analysis of the 3D non-linear Stokes problem coupled to transport-diffusion for shear- thinning heterogeneous microscale flows, applications to digital rock physics and mucociliary clearance," ESAIM: Mathematical Modelling and Numerical Analysis, vol. 53, pp. 1083-1124, 2019.

58. C. Soulaine, S. Roman, A. Kovscek, and H. Tchelepi, "Pore-scale modelling of multiphase reactive flow: application to mineral dissolution with production of $\mathrm{CO}_{2}$," Journal of Fluid Mechanics, vol. 855, p. 616-645, 2018.

59. D. Lasseux, M. Quintard, and S. Whitaker, "Determination of permeability tensors for two-phase flow in homogeneous porous media: Theory," Transport in Porous Media, vol. 24, no. 2, pp. 107-137, 1996-08.

60. A. Q. Raeini, M. J. Blunt, and B. Bijeljic, "Direct simulations of two-phase flow on micro-ct images of porous media and upscaling of pore-scale forces," Advances in Water Resources, vol. 74, pp. 116 - 126, 2014.

61. F. Zami-Pierre, R. Loubens, M. Quintard, and Y. Davit, "Polymer flow through porous media: Numerical prediction of the contribution of slip to the apparent viscosity," Transport in Porous Media, vol. 119, pp. 1-18, 092017.

62. Y. Saad and M. Schultz, "GMRES: a generalized minimal residual method for solving nonsymmetric linear systems.," SIAM Journal on scientific and statistical computing, vol. 7, no. 3, pp. 856-869, 1986. 